\title{
Direct detection and LHC constraints on a $t$-channel simplified model of Majorana dark matter at one loop
}

\author{
Kirtimaan A. Mohan, ${ }^{a}$ Dipan Sengupta, ${ }^{a, b}$ Tim M.P Tait, ${ }^{c}$ Bin Yan $^{a}$ and C.-P. Yuan ${ }^{a}$ \\ ${ }^{a}$ Department of Physics and Astronomy, Michigan State University, \\ 567 Wilson Road, East Lansing, MI, U.S.A \\ ${ }^{b}$ Department of Physics and Astronomy, University of California, San Diego, \\ 9500 Gilman Drive, La Jolla, CA, U.S.A \\ ${ }^{c}$ Department of Physics and Astronomy, University of California, Irvine, \\ Irvine, CA 92697-4575, U.S.A. \\ E-mail: kamohan@pa.msu.edu, disengupta@physics.ucsd.edu, \\ ttait@uci.edu, yanbin1@msu.edu, yuan@pa.msu.edu
}

ABSTRACT: An interesting class of models posits that the dark matter is a Majorana fermion which interacts with a quark together with a colored scalar mediator. Such a theory can be tested in direct detection experiments, through dark matter scattering with heavy nuclei, and at the LHC, via jets and missing energy signatures. Motivated by the fact that such theories have spin-independent interactions that vanish at tree level, we examine them at one loop (along with RGE improvement to resum large logs), and find that despite its occurrence at a higher order of perturbation theory, the spin-independent scattering searches typically impose the strongest constraints on the model parameter space. We further analyze the corresponding LHC constraints at one loop and find that it is important to take them into account when interpreting the implications of searches for jets plus missing momentum on this class of models, thus providing the corresponding complementary information for this class of models.

Keywords: Beyond Standard Model, Cosmology of Theories beyond the SM, Renormalization Group

ARXIV EPRINT: 1903.05650 


\section{Contents}

1 Introduction $\quad 1$

2 Simplified model and parameters 3

3 Scattering with heavy nuclei 4

3.1 Wilson coefficients 7

3.2 Renormalization group evolution and threshold matching 9

$\begin{array}{lll}3.2 .1 & \text { Collinear divergence } & 12\end{array}$

$\begin{array}{lll}3.3 & \text { Limits from direct searches } & 13\end{array}$

$\begin{array}{lll}3.3 .1 & \text { Spin dependent limits } & 13\end{array}$

$\begin{array}{ll}\text { 3.3.2 Spin independent limits } & 13\end{array}$

4 Dark matter production at the LHC $\quad 14$

$\begin{array}{lll}4.1 & \text { Cross-sections at NLO } & 16\end{array}$

$\begin{array}{lll}4.2 & \text { Experimental searches } & 18\end{array}$

$\begin{array}{lll}\text { 4.2.1 } & \text { Mono-jet }+\mathbb{E}_{T} \text { search } & 18\end{array}$

$\begin{array}{lll}4.2 .2 & \text { Multi-jet }+\mathbb{E}_{T} \text { search } & 20\end{array}$

5 Combined limits $\quad 22$

6 Outlook 23

$\begin{array}{lr}\text { A Wilson coefficients } & \mathbf{2 5}\end{array}$

A.1 Quarkionic Wilson coefficients 25

A.2 Gluonic Wilson coefficients 25

A.3 Gluonic Wilson coefficients using the Fock-Schwinger gauge 27

$\begin{array}{lr}\text { B Numerical values } & 28\end{array}$

\section{Introduction}

Observations from cosmology and large scale structures indicate that the Universe is filled with a non-relativistic species of particle that so far appears to be completely transparent to photons of all wavelengths. The properties of this dark matter (DM) appear to be inconsistent with any ingredient known to the Standard Model (SM) of particle physics, and thus represents a glimpse of physics beyond it. If the dark matter has appreciable interaction with the SM fields, its abundance in the Universe today can be understood as the result of a thermal freeze-out process. Based on this hope, there is a major effort 
currently underway to detect it through its annihilation products, scattering with ordinary matter (such as heavy nuclei), or by producing it directly at high energy colliders.

Assembling a complete picture of what the dark matter can (or cannot) be requires us to collate information from all sources. Understanding how to map one class of search into another one requires a theoretical framework. Within the thermal freeze-out paradigm, there are a variety of possible theories of dark matter, ranging in completeness from effective field theories to simplified models to UV complete theories such as supersymmetry. Recently, simplified models have emerged as a robust mechanism to contrast various particle searches, as they balance a reasonable simple theoretical framework with a finite number of parameters against enough detail to encapsulate a theoretically complete description valid up to the energies probed a the Large Hadron Collider (LHC) [1].

There are a variety of simplified models employed to interpret LHC searches, largely classified by the nature of the mediator which communicates between the SM and the dark sectors. Much of previous work has considered "s-channel models" in which the mediator is a dark force carrier, a neutral boson which has interactions both with a pair of dark matter particles and with a pair of Standard Model particles [2-5]. While interesting parameter space remains to be explored, such constructions are generically constrained by searches for visible decays of the mediator $[6,7]$. A different, and equally compelling class of models contains colored mediator particles, which can either interact directly with a quark and a dark matter particle [8-15], or act as a bridge at loop level between a pair of dark matter particles and a pair of gluons [16-18]. Such colored mediators are in principle accessible at the LHC, leading to signatures of missing momentum accompanied by energetic jets of hadrons, and are necessary ingredients in UV complete models of physics beyond the Standard Model such as supersymmetry or Little Higgs models. At the same time, in contrast to the $s$ channel models, there are no purely SM searches to restrict the viable parameter space. They are thus important to understand the relative importance of jets plus missing momentum searches to probe dark matter models. Simultaneously, direct detection experiments also provide strong constraints on the parameter space of these models, thus providing complementary information. Current and next generation direct detection experiments will probe a large part of the Weakly Interacting Massive Particle (WIMP) regime of the dark matter landscape, thus providing a better understanding of the theoretical space of models.

In order to correctly parse the implications for experimental searches on the parameter space of dark matter models, accurate theoretical predictions are required. This is particularly important in the case where the dark matter is a Majorana fermion and the mediator is a colored scalar particle. In that case, the tree level contribution to the spinindependent scattering with nuclei vanishes, leaving much weaker constraints from spindependent searches [11]. But contributions to the spin-independent scattering rate still occur at one loop level, and as we shall see below, represent the dominant constraints for wide regions of parameter space. In this article, we extend our understanding of this simplified model to the next-to-leading-order:

- We compute the one loop (leading non-vanishing) order contribution to the spinindependent scattering operator, and perform renormalization group evolution (RGE) 
from high scales of order the mediator mass down to the low energy scales relevant for dark matter scattering with a heavy nucleus.

- We compute the LHC production cross sections to next-to-leading order (NLO) in $\alpha_{S}$, and recast the existing LHC searches into the simplified model parameter space.

Our results demonstrate that these refinements significantly alter the remaining viable parameter space in light of the null searches for dark matter scattering, and also make important changes to the impact and prospects of searches at the LHC. In particular, we find that the picture based on the leading order scattering changes by roughly an order of magnitude when next-to-leading order contributions are included. The impact on limits from the LHC is less dramatic, but nonetheless DM production rates can change by as much as $\sim 50 \%$, leading to very significant impact on the allowed parameter space.

The rest of this paper is organized as follows. In section 2 we describe the simplified model and the assumptions concerning the parameter space. In section 3, we discuss the scattering with heavy nuclei, including the NLO contributions and RGE evolution of the Wilson coefficients. In section 4, we assess the complementary collider constraints originating from LHC searches. In section 5, we provide a summary of all constraints, as well as compute the velocity averaged annihilation cross section in order to assess whether this class of models can provide the correct thermal relic. Finally we present our concluding remarks in section 6 .

\section{Simplified model and parameters}

In this section, we briefly review the simplified model, more details of which can be found in [11]. The simplified model contains a SM singlet fermionic dark matter candidate $(\chi)$, whose kinetic terms are described by the Lagrangian

$$
\mathcal{L}_{\chi}=\frac{1}{2}\left(i \bar{\chi} \not \partial \chi-M_{\chi} \bar{\chi} \chi\right)
$$

While $\chi$ can be either Dirac or Majorana, we specialize to the Majorana case where large corrections are expected to the cross section for scattering with nuclei. There are also a set of scalar mediator particles, which, to interact with the dark matter and a SM quark, must be color triplets transforming under the electroweak symmetry as (using notation $\left.(\mathrm{SU}(3), \mathrm{SU}(2))_{Y}\right)$ :

$$
(3,1)_{2 / 3}, \quad(3,1)_{-1 / 3}, \quad(3,2)_{-1 / 6}
$$

These three choices correspond to what we will refer to as a $u_{R}$ model (with mediators labeled as $\tilde{u}$ ), a $d_{R}$ model (with mediators $\tilde{d}$ ), and a $q_{L}$ model (with mediators $\tilde{Q}$ ), respectively. Motivated by the assumption of minimal flavor violation (MFV) [19], we assign the mediators to flavor triplets with equal masses and couplings. Thus the mediator and its 


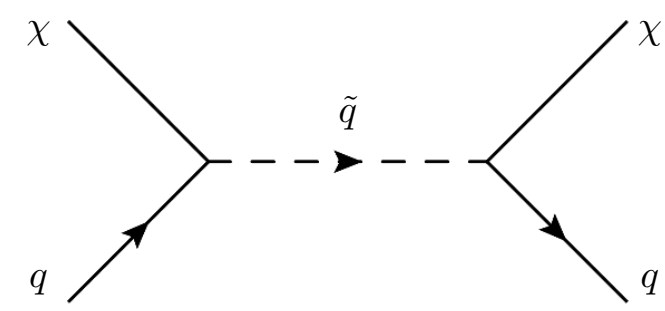

Figure 1. Representative Feynman diagram for scattering between DM and quarks.

dynamics can be described by the corresponding choice of Lagrangian:

$$
\begin{aligned}
& \mathcal{L}_{u_{R}}=\sum_{u}\left[\left(D_{\mu} \tilde{u}\right)^{*}\left(D^{\mu} \tilde{u}\right)-M_{\tilde{u}}^{2} \tilde{u}^{*} \tilde{u}+g_{D M} \tilde{u}^{*} \bar{\chi} P_{R} u+g_{D M}^{*} \tilde{u} \bar{u} P_{L} \chi\right], \\
& \mathcal{L}_{d_{R}}=\sum_{d}\left[\left(D_{\mu} \tilde{d}\right)^{*}\left(D^{\mu} \tilde{d}\right)-M_{\tilde{d}}^{2} \tilde{d}^{*} \tilde{d}+g_{D M} \tilde{d}^{*} \bar{\chi} P_{R} d+g_{D M}^{*} \tilde{d} \bar{d} P_{L} \chi\right], \\
& \left.\mathcal{L}_{q_{L}}=\sum_{q}\left[\left(D_{\mu} \tilde{q}\right)^{*}\left(D^{\mu} \tilde{q}\right)-M_{\tilde{q}}^{2} \tilde{q}^{*} \tilde{q}+g_{D M} \tilde{q}^{*} \bar{\chi} P_{L} q+g_{D M}^{*} \tilde{q} \bar{q} P_{R} \chi\right)\right],
\end{aligned}
$$

where the covariant derivative $D_{\mu}=\left(\partial_{\mu}-i g_{s} G_{\mu}^{a} T^{a}+\right.$ Electroweak terms $)$, describes the mediator couplings to the SM gauge bosons. Here the sums are over quark and mediator flavors where $u=\{u, d, s\}$ quarks, $\tilde{u}=\{\tilde{u}, \tilde{d}, \tilde{s}\}$ mediators, $d=\{d, s, b\}$ quarks, $\tilde{d}=$ $\{\tilde{d}, \tilde{s}, \tilde{b}\}$ mediators, $q=\{u, d, s, c, b, t\}$ quarks and $\tilde{q}=\{\tilde{u}, \tilde{d}, \tilde{s}, \tilde{c}, \tilde{b}, \tilde{t}\}$ mediators. In order to have a dark matter candidate which is uncolored or charged, we restrict ourselves to the parameter space in which all of the mediator masses are larger than $M_{\chi}$.

Generation-dependent masses and couplings that are higher order in the Yukawa couplings can be generated consistently with MFV and can be described (for example, for the $\tilde{u}_{R}$ model) by terms such as:

$$
\left.\mathcal{L}_{F V}=\left(\delta g_{D M} \tilde{u}^{*} Y^{u \dagger} Y^{u} \bar{\chi} P_{R} u+\text { h.c. }\right)+\delta m^{2} \tilde{u}^{*} Y^{u \dagger} Y^{u} \tilde{u}+\mathcal{O}\left(Y^{4}\right) .\right),
$$

where $Y^{u}$ is the SM Yukawa matrix. For simplicity and to avoid potential strong constraints from the null results of searches for flavor and CP-violation, we take $\delta g_{D M}=\delta m^{2}=0$ and choose $g_{D M}$ to be real, from here onward.

\section{Scattering with heavy nuclei}

In the non-relativistic limit, dark matter scattering with a nucleus is described by a spinindependent (SI) term, which at low momentum transfer resolves the entire nucleus coherently leading to a cross section enhanced by the squared number of scattering centers (nucleons); and a spin-dependent (SD) term, which couples to the nucleon spin and typically enjoys no coherent enhancement for large nuclei.

We begin by reviewing some of the results of Reference [11], which represents the baseline upon which our improvements build. At leading order (LO), the dark matter interacts with a generic quark $q$ via tree level exchange of its corresponding mediator $\tilde{q}$, 
as shown in a representative Feynman diagram in figure 1. The partonic matrix element for interactions between fermionic (Dirac or Majorana) dark matter and up-type quarks mediated by $\tilde{u}$ takes the form,

$$
\begin{aligned}
\mathcal{M} & =\left(-i g_{D M}\right)^{2}\left(\bar{\chi} P_{R} u\right) \frac{i}{p^{2}-M_{\tilde{u}}^{2}}\left(\bar{u} P_{L} \chi\right) \\
\approx & \left(-i g_{D M}\right)^{2}\left(\bar{\chi} P_{R} u\right) \frac{-i}{M_{\tilde{u}}^{2}-m_{\chi}^{2}}\left(\bar{u} P_{L} \chi\right)+\mathcal{O}\left(\left[\frac{1}{M_{\tilde{u}}^{2}-m_{\chi}^{2}}\right]^{2}\right) \\
= & \frac{i g_{D M}^{2}}{M_{\tilde{u}}^{2}-m_{\chi}^{2}} \frac{1}{8}\left[\left(\bar{\chi} \gamma^{\mu} \chi\right)\left(\bar{u} \gamma_{\mu} u\right)-\left(\bar{\chi} \gamma^{\mu} \gamma^{5} \chi\right)\left(\bar{u} \gamma_{\mu} \gamma_{5} u\right)\right. \\
& \left.\quad+\left(\bar{\chi} \gamma^{\mu} \gamma^{5} \chi\right)\left(\bar{u} \gamma_{\mu} u\right)-\left(\bar{\chi} \gamma^{\mu} \chi\right)\left(\bar{u} \gamma_{\mu} \gamma_{5} u\right)\right] \\
\approx & \frac{i g_{D M}^{2}}{M_{\tilde{u}}^{2}-m_{\chi}^{2}} \frac{1}{8}\left[\left(\bar{\chi} \gamma^{\mu} \chi\right)\left(\bar{u} \gamma_{\mu} u\right)-\left(\bar{\chi} \gamma^{\mu} \gamma^{5} \chi\right)\left(\bar{u} \gamma_{\mu} \gamma_{5} u\right)\right]
\end{aligned}
$$

where, in the second line, the propagator is expanded in the low momentum limit and only leading terms are kept. As discussed in section 3.1 and appendix A, higher order terms (which were dropped in Reference [11]) turn out to be important. In the last line of equation (3.1), we have dropped terms which are negligible in the non-relativistic limit. Furthermore, we have dropped the quark mass from the expressions above to simplify them. Majorana fermions are treated using the technology of refs. [20, 21]. Analogous results as above hold for $d_{R}$ and $q_{L}$ quarks mediated by $\tilde{d}$ and $\tilde{q}$, respectively. The terms in the last line result in spin independent and spin dependent scattering, respectively. However, since a Majorana fermion has a vanishing vector bilinear $\left(\bar{\chi} \gamma^{\mu} \chi=0\right)$, only the SD terms are non-zero at this order. ${ }^{1}$ In order to assess the rate of SI scattering, it is necessary to go beyond the simple leading order calculation.

Following the notation of refs. [22] and [23] we write down the lagrangian for the effective field theory describing SI interactions with quarks and gluons,

$$
\mathcal{L}_{S I}^{\mathrm{eff}}=\sum_{q=u, d, s} \mathcal{L}_{q}^{\mathrm{eff}}+\mathcal{L}_{g}^{\mathrm{eff}}
$$

where

$$
\begin{aligned}
& \mathcal{L}_{q}^{\mathrm{eff}}=f_{q} \bar{\chi} \chi O_{q}^{(0)}+\frac{g_{q}^{(1)}}{m_{\chi}} \bar{\chi} i\left(\partial^{\mu} \gamma^{\nu}+\partial^{\nu} \gamma^{\mu}\right) \chi O_{q, \mu \nu}^{(2)}+\frac{g_{q}^{(2)}}{m_{\chi}^{2}} \bar{\chi}\left(i \partial^{\mu}\right)\left(i \partial^{\nu}\right) \chi O_{q, \mu \nu}^{(2)} \\
& \mathcal{L}_{g}^{\mathrm{eff}}=f_{G} \bar{\chi} \chi O_{g}^{(0)}+\frac{g_{G}^{(1)}}{m_{\chi}} \bar{\chi} i\left(\partial^{\mu} \gamma^{\nu}+\partial^{\nu} \gamma^{\mu}\right) \chi O_{g, \mu \nu}^{(2)}+\frac{g_{G}^{(2)}}{m_{\chi}^{2}} \bar{\chi}\left(i \partial^{\mu}\right)\left(i \partial^{\nu}\right) \chi O_{g, \mu \nu}^{(2)}
\end{aligned}
$$

\footnotetext{
${ }^{1}$ It is worth noting that this feature is a consequence of having a single type of mediator. In theories with both $\tilde{Q}$ and either $\tilde{u}$ or $\tilde{d}$ type mediators, there may be renormalizable interactions involving both mediators and a Higgs boson, which would open up the possibility for tree level spin-independent scattering.
} 
and the SI operators

$$
\begin{array}{rlrl}
O_{q}^{(0)} & \equiv m_{q} \bar{q} q, & O_{q}^{(2) \mu \nu} \equiv \frac{1}{2} \bar{q}\left(\gamma^{\{\mu} i D_{-}^{\nu\}}-\frac{g^{\mu \nu}}{4} i \not D_{-}\right) q \\
O_{g}^{(0)} & \equiv G_{\mu \nu}^{A} G^{A \mu \nu}, & O_{g}^{(2) \mu \nu} & \equiv-G^{A \mu \lambda} G_{\lambda}^{A \nu}+\frac{g^{\mu \nu}}{4}\left(G_{\alpha \beta}^{A}\right)^{2} .
\end{array}
$$

The standard shorthand notation used in the above expressions read as,

$$
\begin{aligned}
A^{\{\mu} B^{\nu\}} & =\left(A^{\mu} B^{\nu}+A^{\nu} B^{\mu}\right) / 2 \\
D_{ \pm}^{\mu} & =D^{\mu} \pm \overleftarrow{D}^{\mu} \\
D_{\mu} & =\partial_{\mu}-i g A_{\mu}^{A} T^{A}-i e Q A_{\mu}^{A} \\
\overleftarrow{D}_{\mu} & =\overleftarrow{\partial}_{\mu}+i g A_{\mu}^{A} T^{A}+i e Q A_{\mu}^{A}
\end{aligned}
$$

The quantities $f_{q}, g_{q}^{(1)}$ and $g_{q}^{(2)}$ are Wilson coefficients generated by matrix elements with quarks in the initial and final states, whereas $f_{G}, g_{G}^{(1)}$ and $g_{G}^{(2)}$ are Wilson coefficients generated by matrix elements with gluons in the initial and final states. Although the operators listed above do not form a complete basis, they are the set of operators that are relevant and sizable for SI nuclear matrix elements.

In this language, the matrix element for dark matter participating in SI scattering elastically with a target nucleon $(N=\{p, n\})$ is [24],

$$
\begin{aligned}
f_{N} / m_{N}= & \sum_{q=u, d, s} f_{T q} f_{q}+\sum_{q=u, d, s, c, b} \frac{3}{4}[q(2)+\bar{q}(2)]\left(g_{q}^{(1)}+g_{q}^{(2)}\right) \\
& -\frac{8 \pi}{9 \alpha_{s}} f_{T_{G}} f_{G}+\frac{3}{4} G(2)\left(g_{G}^{(1)}+g_{G}^{(2)}\right),
\end{aligned}
$$

where $m_{N}$ is the mass of the nucleon and $f_{T q}, f_{T G}, q(2), \bar{q}(2)$ and $G(2)$ represent hadronic matrix elements:

$$
\begin{aligned}
\left\langle N\left|m_{q} \bar{q} q\right| N\right\rangle / m_{N} & \equiv f_{T q}, \\
\left\langle N\left|-\frac{9 \alpha_{s}}{8 \pi} G_{\mu \nu}^{A} G^{A \mu \nu}\right| N\right\rangle / m_{N} & \equiv f_{T_{G}} \\
\left\langle N(p)\left|\mathcal{O}_{q, \mu \nu}^{(2)}\right| N(p)\right\rangle & =\frac{1}{m_{N}}\left(p_{\mu} p_{\nu}-\frac{1}{4} m_{N}^{2} g_{\mu \nu}\right)[q(2)+\bar{q}(2)], \\
\left\langle N(p)\left|\mathcal{O}_{g, \mu \nu}^{(2)}\right| N(p)\right\rangle & =\frac{1}{m_{N}}\left(p_{\mu} p_{\nu}-\frac{1}{4} m_{N}^{2} g_{\mu \nu}\right) G(2) .
\end{aligned}
$$

The matrix elements of the light quarks $(q=u, d, s)$ are determined from lattice calculations of the pion nucleon sigma term,

$$
\begin{aligned}
\Sigma_{\pi N} & =\frac{m_{u}+m_{d}}{2}\langle N|(\bar{u} u+\bar{d} d)| N\rangle, \\
\Sigma_{-} & =\left(m_{d}-m_{u}\right)\langle N|(\bar{u} u-\bar{d} d)| N\rangle .
\end{aligned}
$$


And the matrix elements of the twist- 2 operators are related to the second moments of the parton distribution functions (PDFs):

$$
\begin{aligned}
{[q(2)+\bar{q}(2)] } & =\int_{0}^{1} d x x[q(x)+\bar{q}(x)], \\
G(2) & =\int_{0}^{1} d x x g(x),
\end{aligned}
$$

where $q(x), \bar{q}(x)$ and $g(x)$ are the PDFs of quarks, anti-quarks and gluons in $N$, respectively. We provide numerical values for the hadronic matrix elements in appendix B.

\subsection{Wilson coefficients}

The Wilson coefficients are determined by matching to matrix elements computed in the simplified model. In this section, we perform this matching at scales of order the mediator mass.

The leading contributions to the quark Wilson coefficients $f_{q}, g_{q}^{(1)}$ and $g_{q}^{(2)}$ arise from the tree level diagrams of figure 1, but at a higher order in expansion of the propagator. For a single flavor of quark with mass $m_{q}$ and its corresponding mediator $\tilde{q}$ of mass $M_{\tilde{q}}$ (and denoted as $M$ in shorthand), the Wilson coefficients are

$$
\begin{aligned}
f_{q} & =\frac{g_{D M}^{2} m_{\chi}}{16\left(M^{2}-m_{\chi}^{2}\right)^{2}}, \\
g_{q}^{(1)} & =\frac{g_{D M}^{2} m_{\chi}}{8\left(M^{2}-m_{\chi}^{2}\right)^{2}}, \\
g_{q}^{(2)} & =0 .
\end{aligned}
$$

Compared to the SD matrix elements in equation (3.1), these Wilson coefficients are suppressed by an additional power of $1 /\left(M^{2}-m_{\chi}^{2}\right)$. Details of the calculation can be found in appendix A. We have ommitted the quark mass from the denominators in the expressions above, but use it in our numerical calculations.

The leading contribution to the gluonic Wilson coefficients arise at one loop, with representative Feynman diagrams shown in figure 2 . The individual Wilson coefficients are extracted using projection operators, with detailed results relegated to appendix A.2. In the limit of small quark mass $\left(m_{q} \rightarrow 0\right)$,

$$
\begin{aligned}
f_{G} & \simeq \frac{\alpha_{s} g_{D M}^{2} m_{\chi}}{192 \pi} \frac{\left(m_{\chi}^{2}-2 M^{2}\right)}{M^{2}\left(M^{2}-m_{\chi}^{2}\right)^{2}} \\
\frac{g_{G}^{(2)}}{m_{\chi}^{2}} & \simeq \alpha_{s} g_{D M}^{2} \frac{-2 M^{2} m_{\chi}^{2}+2\left(M^{2}-m_{\chi}^{2}\right)^{2} \log \left(\frac{M^{2}}{M^{2}-m_{\chi}^{2}}\right)+3 m_{\chi}^{4}}{48 \pi m_{\chi}^{5}\left(M^{2}-m_{\chi}^{2}\right)^{2}} \\
\frac{g_{G}^{(1)}}{m_{\chi}} & \simeq \frac{\alpha_{s} g_{D M}^{2}}{96 \pi m_{\chi}^{4}\left(M^{2}-m_{\chi}^{2}\right)^{2}}\left[-2 m_{\chi}^{4} \log \left(\frac{m_{q}^{2}}{M^{2}}\right)-m_{\chi}^{2}\left(M^{2}+3 m_{\chi}^{2}\right)\right. \\
& \left.+\left(M^{2}-3 m_{\chi}^{2}\right)\left(M^{2}+m_{\chi}^{2}\right) \log \left(\frac{M^{2}}{M^{2}-m_{\chi}^{2}}\right)\right]
\end{aligned}
$$



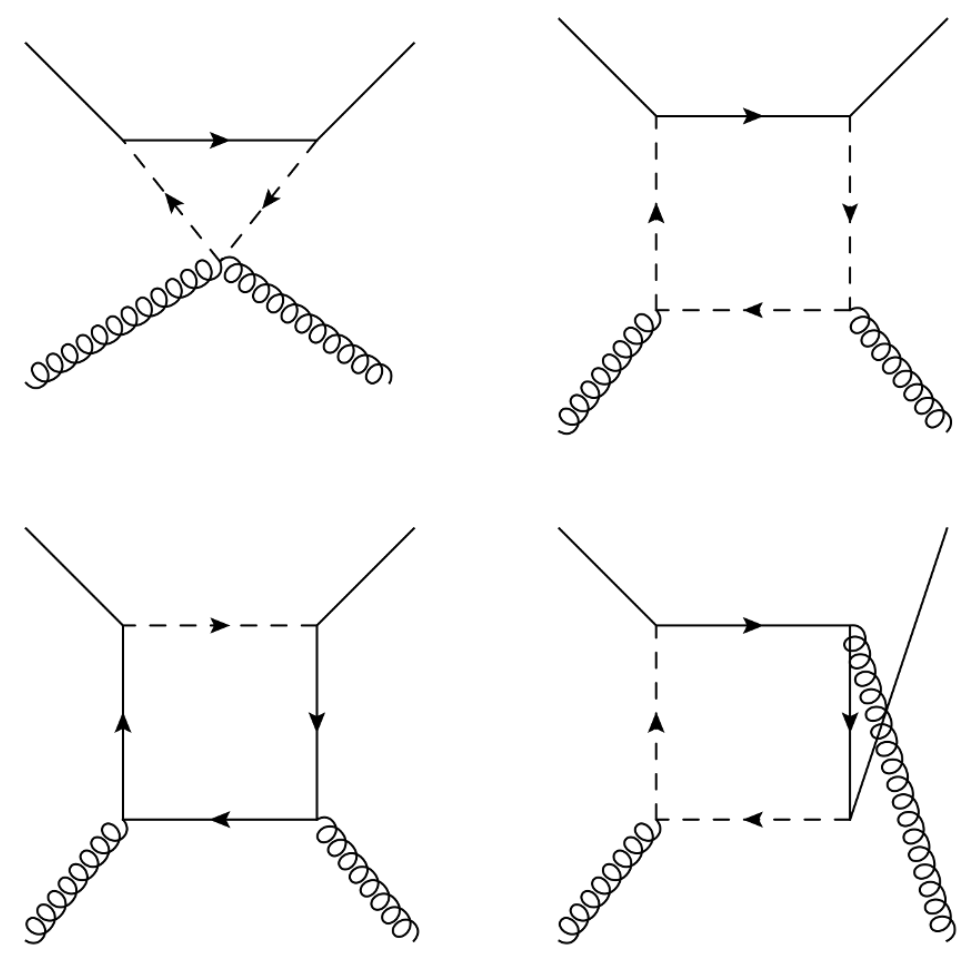

Figure 2. Representative Feynman diagrams for DM scattering with gluons.

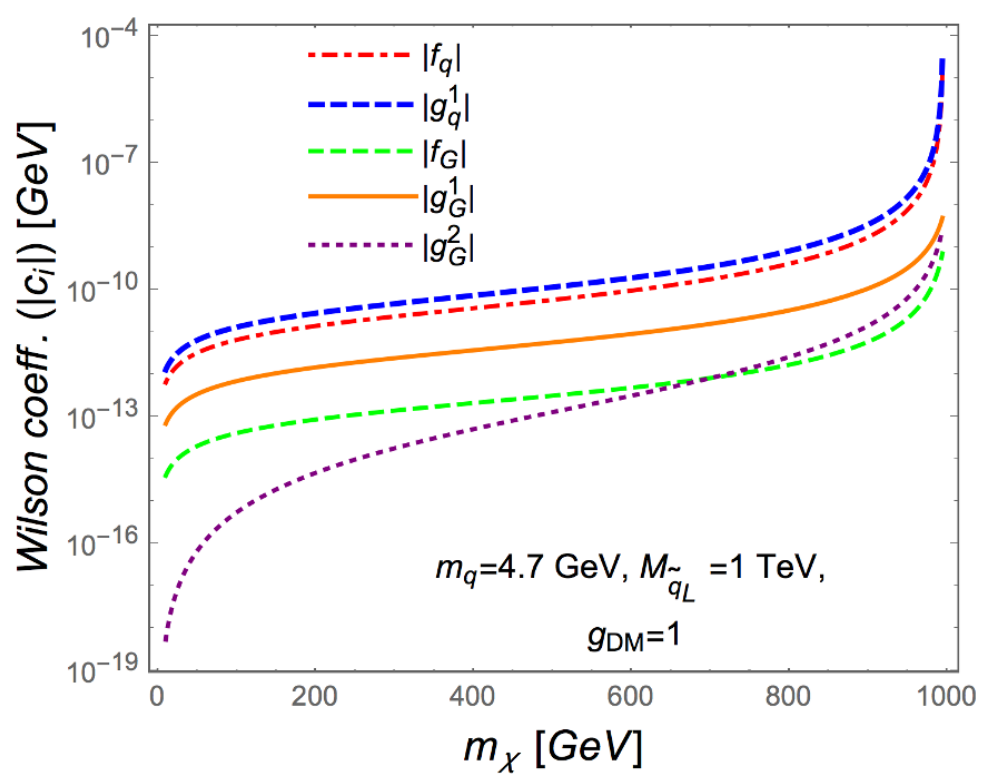

Figure 3. Absolute values of the bottom quark and gluon Wilson coefficients (as indicated, in appropriate powers of $\mathrm{GeV}$ for each) as a function of the dark matter mass, for $M_{\tilde{q}}=1 \mathrm{TeV}$, $m_{q}=m_{b}=4.2 \mathrm{GeV}$ and $g_{D M}=1$.

They arise at the same power of $1 /\left(M^{2}-m_{\chi}^{2}\right)$, but are suppressed by $\alpha_{s}$ as compared to the corresponding quark SI Wilson coefficients, cf eq. (3.11). 
In figure 3 we show the absolute value of the bottom quark (with $m_{q}=m_{b}=4.2 \mathrm{GeV}$ ) and gluon SI Wilson coefficients as a function of the dark matter mass, and for a representative parameter point with $M_{\tilde{q}}=1 \mathrm{TeV}$ and $g_{D M}=1$. Each coefficient is expressed in units of $\mathrm{GeV}$ to the appropriate power. All of the Wilson coefficients have a resonant enhancement in the limit $m_{\chi} \rightarrow M_{\tilde{q}}$. We observe that the gluonic Wilson coefficients are roughly an order of magnitude smaller than their quark counterparts. However, this feature is mitigated by the fact that their hadronic matrix elements are large and RGE effects are important, especially for the spin-0 gluonic term $\mathcal{O}_{g}^{(0)}$.

We note that while the quark coefficients $f_{q}$ and $g_{q}^{(1)}$ are independent of the quark mass at this order, the gluonic coefficients $f_{G}, g_{G}^{(1)}$ and $g_{G}^{(2)}$ all depend on the mass of the quark in the loop. In the limit of $m_{q} \rightarrow 0, f_{G}$ and $g_{G}^{(2)}$ reduce to the finite expressions in equations (3.12) and (3.13). In contrast, as indicated in equation (3.14), $g_{G}^{(1)}$ diverges logarithmically with divergent piece:

$$
\Delta g_{G}^{(1)}=\frac{\alpha_{s} g_{D M}^{2} m_{\chi}}{24 \pi\left(M^{2}-m_{\chi}^{2}\right)^{2}} \log \left(\frac{M}{m_{q}}\right)=g_{q}^{(1)} \frac{\alpha_{s}}{3 \pi} \log \left(\frac{M}{m_{q}}\right) .
$$

From the second expression, we observe that it can be rewritten in terms of the quark Wilson coefficient $g_{q}^{(1)}$, suggesting that it might cancel against the $\alpha_{s}$ real correction to the quark scattering. We demonstrate below in the context of the renormalization group evolution that this is indeed the case.

\subsection{Renormalization group evolution and threshold matching}

The Wilson coefficients are matched to the simplified model at a scale $\mu \simeq M_{\tilde{q}}$ which is appropriate to describe production of the mediators at the LHC. To make accurate predictions at the low energy scales appropriate for direct detection, we evolve them to $\mu_{l}=2 \mathrm{GeV}$ via renormalization group equations, which we evaluate at leading log in the strong coupling $\alpha_{s}$, following refs. [23, 25, 26]. We neglect subleading corrections from the electroweak interactions.

The strong force corrections to the SI operators boil down to the corrections to the quark and gluon bilinear factors $O_{q}^{(0)}, O_{q}^{(2) \mu \nu}, O_{g}^{(0)}$, and $O_{g}^{(2) \mu \nu}$ in the SI EFT Lagrangian ${ }^{2}$ of equation (3.4), equation (3.4). Under the renormalization group, the operators $O_{i}$ and their Wilson coefficients $c_{i}$ evolve according to their anomalous dimensions $\gamma_{i j}$ :

$$
\frac{d}{d \log \mu} O_{i}=-\gamma_{i j} O_{j}, \quad \frac{d}{d \log \mu} c_{i}=\gamma_{j i} c_{j} .
$$

The solution evolving from a high scale $\mu_{h}$ down to a low scale $\mu_{l}$ takes the form:

$$
c_{i}\left(\mu_{l}\right)=R_{i j}\left(\mu_{l}, \mu_{h}\right) c_{j}\left(\mu_{h}\right),
$$

where $R$ is a square matrix in flavor space and $c_{i}$ and $c_{j}$ are column vectors of Wilson coefficients arranged in flavor space as $c_{j}=(u, d, s, c, b, t \mid g)$. Conservation of angular

\footnotetext{
${ }^{2}$ The SD EFT operators contain $V_{q}^{\mu}=\bar{q} \gamma^{\mu} q$ and $A_{q}^{\mu}=\bar{q} \gamma^{\mu} \gamma_{5} q$, which do not receive large RGE corrections.
} 
momentum forbids mixing between the scalar (0) and tensor (2) operators, allowing us to consider them in two separate groups. For each group, the matrix $R^{(i=0,2)}$ is square in flavor space with $n_{f}$ quark flavors:

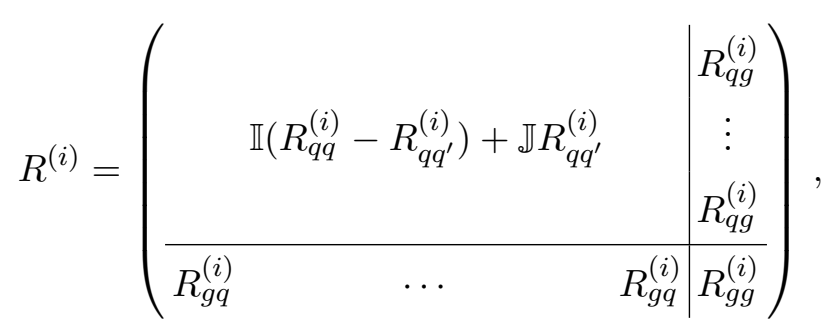

where the $n_{f} \times n_{f}$ matrices $\mathbb{I}$ and $\mathbb{J}$ are the identity matrix and the matrix with all elements equal to unity, respectively. The upper $n_{f} \times n_{f}$ block diagonal entries describe mixing among the quark flavors, and lower diagonal entry renormalize the gluonic operator. The block-off-diagonal terms induce mixing between the quark and gluon operators. For the scalar operators [26]:

$$
\begin{array}{ll}
R_{q q}^{(0)}=1, & R_{q g}^{(0)}=2\left[\gamma_{m}\left(\mu_{h}\right)-\gamma_{m}\left(\mu_{l}\right)\right] / \tilde{\beta}\left(\mu_{h}\right), \\
R_{q q^{\prime}}^{(0)}=R_{g q}^{(0)}=0, & R_{g g}^{(0)}=\tilde{\beta}\left(\mu_{l}\right) / \tilde{\beta}\left(\mu_{h}\right),
\end{array}
$$

where $\beta$ is the QCD beta function and $\tilde{\beta} \equiv \beta / g_{s}$. At this order, there is no mixing between operators of different quark flavors. The form of the quark/gluon mixing can be understood as a sum rule that determines the quark and gluon contributions to the mass of the nucleon. For the spin-2 operators [26]:

$$
\begin{array}{rlrl}
R_{q q}^{(2)} & =R_{q q^{\prime}}^{(2)}+r(0), & R_{q q^{\prime}}^{(2)} & =\frac{1}{n_{f}}\left[\frac{16 r\left(n_{f}\right)+3 n_{f}}{16+3 n_{f}}-r(0)\right], \\
R_{q g}^{(2)} & =\frac{16\left[1-r\left(n_{f}\right)\right]}{16+3 n_{f}}, & R_{g q}^{(2)}=\frac{3\left[1-r\left(n_{f}\right)\right]}{16+3 n_{f}}, \\
R_{g g}^{(2)}=\frac{16+3 n_{f} r\left(n_{f}\right)}{16+3 n_{f}}, &
\end{array}
$$

where

$$
r(t) \equiv\left(\frac{\alpha_{s}\left(\mu_{l}\right)}{\alpha_{s}\left(\mu_{h}\right)}\right)^{-\frac{1}{2 \beta_{0}}\left(\frac{64}{9}+\frac{4}{3} t\right)},
$$

with $\beta_{0}=11-2 / 3 n_{f}$. For the spin- 2 operators, there is mixing both between quark flavors and between quarks and the gluons. They are governed by sum rules of PDFs in nucleons.

At the threshold scale for each heavy quark $\mu=m_{Q}$, the heavy quark is integrated out pertrubatively and the Wilson coefficients in the $n_{f}+1$ flavor theory are matched to the $n_{f}$ flavor theory:

$$
c_{i}^{\left(n_{f}\right)}\left(\mu_{Q}\right)=M_{i j}\left(\mu_{Q}\right) c_{j}^{\left(n_{f}+1\right)}\left(\mu_{Q}\right),
$$




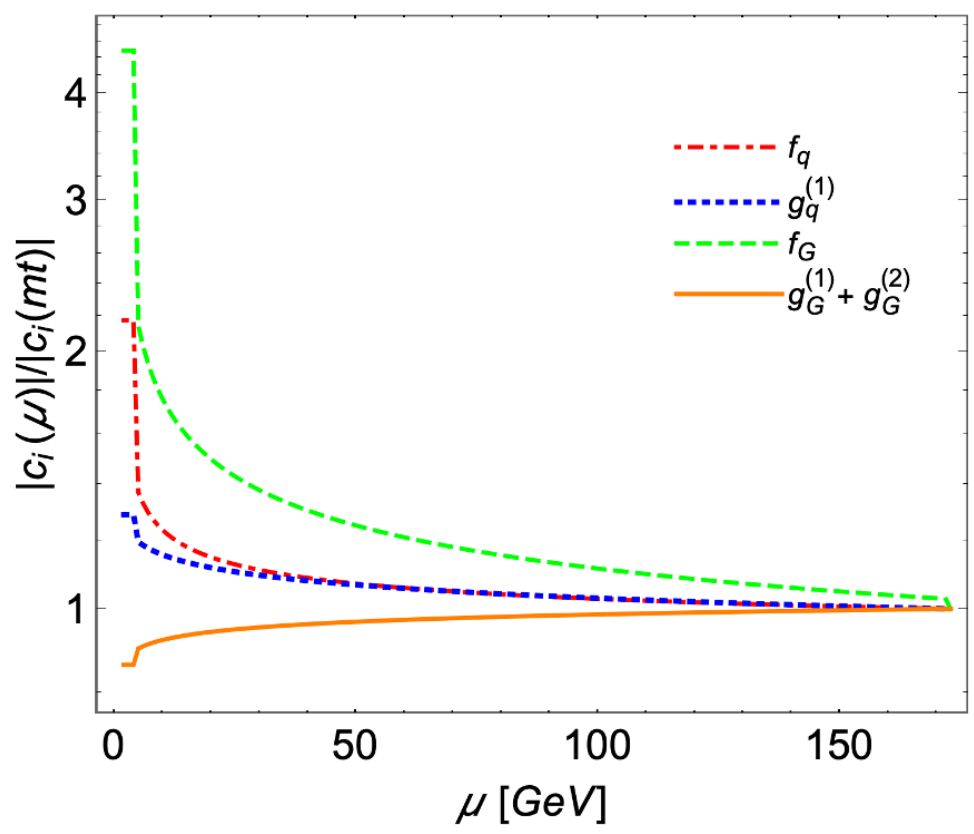

Figure 4. Variation of the $\log _{10}$ ratio of Wilson coefficients $c_{i}(\mu) / c_{i}\left(m_{t}\right)$, as indicated, with the scale $\mu$, for the $Q_{L}$ model with $m_{\chi}=10 \mathrm{GeV}, M_{\tilde{q}}=1 \mathrm{TeV}$, and $g_{D M}=1$.

where $M_{i j}$ is the rectangular matrix

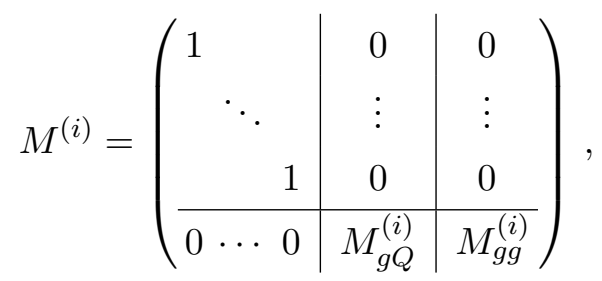

and $n_{f}$ denotes the number of light quark flavors with quark mass less than the energy scale $\mu_{Q}$. The entries of the matching matrix for the spin-0 operators are [26]:

$$
\begin{aligned}
& M_{g Q}^{(0)}=-\frac{\alpha_{s}^{\prime}\left(\mu_{Q}\right)}{12 \pi}\left\{1+\frac{\alpha_{s}^{\prime}\left(\mu_{Q}\right)}{4 \pi}\left[11-\frac{4}{3} \log \frac{\mu_{Q}}{m_{Q}}\right]+\mathcal{O}\left(\alpha_{s}^{2}\right)\right\}, \\
& M_{g g}^{(0)}=1-\frac{\alpha_{s}^{\prime}\left(\mu_{Q}\right)}{3 \pi} \log \frac{\mu_{Q}}{m_{Q}}+\mathcal{O}\left(\alpha_{s}^{2}\right) .
\end{aligned}
$$

Here $\alpha_{s}^{\prime}$ denotes the strong coupling in the $n_{f}+1$ flavor theory. The elements of the matching matrix for spin-2 operators are [26]:

$$
\begin{aligned}
M_{g Q}^{(2)} & =\frac{\alpha_{s}^{\prime}}{3 \pi} \log \frac{\mu_{Q}}{m_{Q}}+\mathcal{O}\left(\alpha_{s}^{2}\right), \\
M_{g g}^{(2)} & =1+\mathcal{O}\left(\alpha_{s}\right) .
\end{aligned}
$$

By matching at the scale $m_{Q}$, only $M_{g Q}^{(0)}$ results in a non-trivial correction.

In figure 4, we show the ratio of the Wilson coefficients to their values at the top quark mass, $c_{i}^{\left(n_{f}\right)}(\mu) / c_{i}^{(6)}\left(m_{t}\right)$, as a function of scale for the $Q_{L}$ model with $m_{\chi}=10 \mathrm{GeV}$, 
$M_{\tilde{q}}=1 \mathrm{TeV}$ and $g_{D M}=1$. At the lowest scale we consider, $\mu=2 \mathrm{GeV}$, the largest impact is on the spin- 0 operators, whose Wilson coefficients change by a factor of $\sim 2-5$, with a more modest impact on the spin- 2 operators $-g_{q}^{(1)}$ increases by a factor of $\sim 1.1$ whereas $\left(g_{G}^{(1)}+g_{G}^{(2)}\right)$ reduces by a small factor of $\sim 0.87$. We have left out $g_{q}^{(2)}$, since it is zero, and we group the gluonic twist-2 Wilson coefficients together, since they appear as sums in the nucleon matrix element in equation (3.7). Although both $f_{G}$ and $f_{q}$ increase at low scales, since the hadronic matrix element for $f_{q}$ is much smaller compared to the other terms, it does not have much impact on the total nucleon dark matter scattering amplitude. The net effect on the amplitude is that, at low scales, it increases roughly by a factor of $\sim 2$ when compared to high scales.

\subsubsection{Collinear divergence}

We observed above that the one loop Wilson coefficient $g_{G}^{(1)}$ contains a collinear divergence, equation (3.15). This divergence is canceled to order $\alpha_{s}$ by the RGE contribution to $g_{G}^{(1)}$ from $g_{q}^{(1)}$. We illustrate how this works for the bottom quark contribution in the $d_{R}$ model when the energy scale reduces from $\mu_{h}$ to $\mu_{l}$ with $\mu_{h}>m_{b} .{ }^{3}$ To expand the RGE contribution, we note that the ratio $\alpha_{s}\left(\mu_{h}\right) / \alpha_{s}\left(\mu_{l}\right)$ can be written as:

$$
\frac{\alpha_{s}\left(\mu_{h}\right)}{\alpha_{s}\left(\mu_{l}\right)}=1+\frac{\alpha_{s}\left(\mu_{h}\right) \beta_{0}}{2 \pi} \log \left[\frac{\mu_{l}}{\mu_{h}}\right]
$$

where $\beta_{0}=11-2 / 3 n_{f}$, which implies that the factor $r(t)$ in the RGE is

$$
r(t)=\left(\frac{\alpha_{s}\left(\mu_{l}\right)}{\alpha_{s}\left(\mu_{h}\right)}\right)^{-\frac{1}{2 \beta_{0}}\left(\frac{64}{9}+\frac{4}{3} t\right)} \simeq 1+\frac{\left(\frac{64}{9}+\frac{4}{3} t\right) \alpha_{s}\left(\mu_{h}\right)}{4 \pi} \log \left[\frac{\mu_{l}}{\mu_{h}}\right] .
$$

Expanding the RGE contribution to $g_{G}^{(1)}$ from $g_{q}^{(1)}$ and combining with the collinear divergent term equation (3.15) yields:

$$
\begin{aligned}
\left.\Delta g_{G}^{(1)}\right|_{\mu_{l}} \simeq \frac{m_{\chi} g_{D M}^{2}}{72 \pi^{2}\left(M_{\tilde{q}}^{2}-m_{\chi}^{2}\right)^{2}} & {\left[3 \pi \alpha_{s}\left(\mu_{h}\right) \log \left(\frac{\mu_{l}}{\mu_{h}}\right)\right.} \\
& \left.+\alpha_{s}\left(M_{\tilde{q}}\right) \log \left(\frac{M_{\tilde{q}}}{m_{b}}\right)\left(3 \pi-5 \alpha_{s}\left(\mu_{h}\right) \log \left(\frac{\mu_{l}}{\mu_{h}}\right)\right)\right] .
\end{aligned}
$$

To order $\alpha_{s}$, the collinear logs cancel provided one chooses $\mu_{h}=M_{\tilde{q}}$ and $\mu_{l}=\mu_{b}$. This procedure removes the large log dependence for the heavy quarks. For the light quarks $(u, d, s)$, whose masses are below the hadronic matching scale $\mu_{l}=2 \mathrm{GeV}$, the cancellation works as outlined above down to $\mu_{l}=2 \mathrm{GeV}$, with the remaining portion fo the divergence being absorbed into their $\overline{\mathrm{MS}}$ masses at that scale.

\footnotetext{
${ }^{3}$ This works for all the $q_{L}$ and $u_{R}$ models as well, where the first threshold occurs at the top quark mass. For the $d_{R}$ model the first threshold in the wilson coefficients occurs at $\mu=m_{b}$ and the usual threshold at $\mu=m_{t}$ still exists in the strong coupling $\alpha_{s}$.
} 


\subsection{Limits from direct searches}

\subsubsection{Spin dependent limits}

The SD cross section is dominated by its tree level contribution at leading order in the $1 / M_{\tilde{q}}$ expansion. A detailed discussion of the matching to the hadronic EFT can be found in ref. [27], ands results in the SD cross sections for the $u_{R}, d_{R}$, and $Q_{L}$ models [11]:

$$
\begin{aligned}
\sigma_{S D}^{u_{R}} & =\frac{3}{16 \pi} \frac{m_{N}^{2} M_{\chi}^{2}}{\left(m_{N}+M_{\chi}\right)^{2}} \frac{g_{D M}^{4}}{\left(M_{\tilde{d}}^{2}-M_{\chi}^{2}\right)^{2}}\left(\Delta u^{N}\right)^{2}, \\
\sigma_{S D}^{d_{R}} & =\frac{3}{16 \pi} \frac{m_{N}^{2} M_{\chi}^{2}}{\left(m_{N}+M_{\chi}\right)^{2}} \frac{g_{D M}^{4}}{\left(M_{\tilde{d}}^{2}-M_{\chi}^{2}\right)^{2}}\left(\Delta d^{N}+\Delta s^{N}\right)^{2}, \\
\sigma_{S D}^{q_{L}} & =\frac{3}{16 \pi} \frac{m_{N}^{2} M_{\chi}^{2}}{\left(m_{N}+M_{\chi}\right)^{2}} \frac{g_{D M}^{4}}{\left(M_{\tilde{d}}^{2}-M_{\chi}^{2}\right)^{2}}\left(\Delta u^{N}+\Delta d^{N}+\Delta s^{N}\right)^{2},
\end{aligned}
$$

where $\Delta u^{N}, \Delta d^{N}$ and $\Delta s^{N}$ are matrix elements, whose values are tabulated in appendix B. Because the spin of a heavy nucleus is typically dominated by a single unpaired nucleon, various direct search experiments are typically more sensitive to either scattering with a proton or a neutron, depending on the target nucleus. Currently, the best SD limits on SD proton scattering for $m_{\chi} \gtrsim 3.5 \mathrm{GeV}$ are from PICO-60 [28] (and from CDMSlite, below that [29]), and the best limits on SD neutron scattering are from LUX [30]. For the all three of the simplified models under consideration, the most stringent constraints are from $\mathrm{SD}$ proton scattering. In figure 5 we show the constraints that arise from the PICO-60 limits for each of the three simplified models, in the plane of the dark matter and mediator masses, with the colored shading representing the upper limit on $g_{D M}$ consistent with the null search results. White regions indicate where the mediator mass is smaller than the dark matter mass. Over-all, the constraints are very weak, $g_{D M} \lesssim 5$, generically allowing any perturbative value of $g_{D M}$ for mediator masses greater than a couple of $\mathrm{TeV}$, although they are somewhat stronger in the resonant region $M_{\tilde{q}} \sim m_{\chi}$ and for small dark matter mass.

\subsubsection{Spin independent limits}

The cross section for SI scattering with a nucleon is expressed in terms of the form factors $f_{N}=\left\{f_{p}, f_{n}\right\}$ as:

$$
\sigma_{S I}^{N}=\frac{4}{\pi}\left(\frac{m_{\chi} m_{N}}{M+m_{N}}\right)^{2}\left|f_{N}\right|^{2}
$$

where $f_{N}$ is related to the Wilson coefficients via equation (3.7). We find that comparing the RGE-improved to the non-RGE-improved results for a typical point in parameter space, the RGE-improved $f_{N}$ are generally about a factor of $\sim 1.9$ larger than the non-RGEimproved results. This translates into an increase of about a factor of $\sim 4$ in the SI cross section, and highlights the importance of the higher order terms to accurately assess $\sigma_{S I}$ in this simplified model.

Experiments sensitive to SI scattering typically unfold the dominant nuclear physics to place limits on $\sigma_{S I}^{N}$, with the best current limits for masses above a few $\mathrm{GeV}$ obtained from 


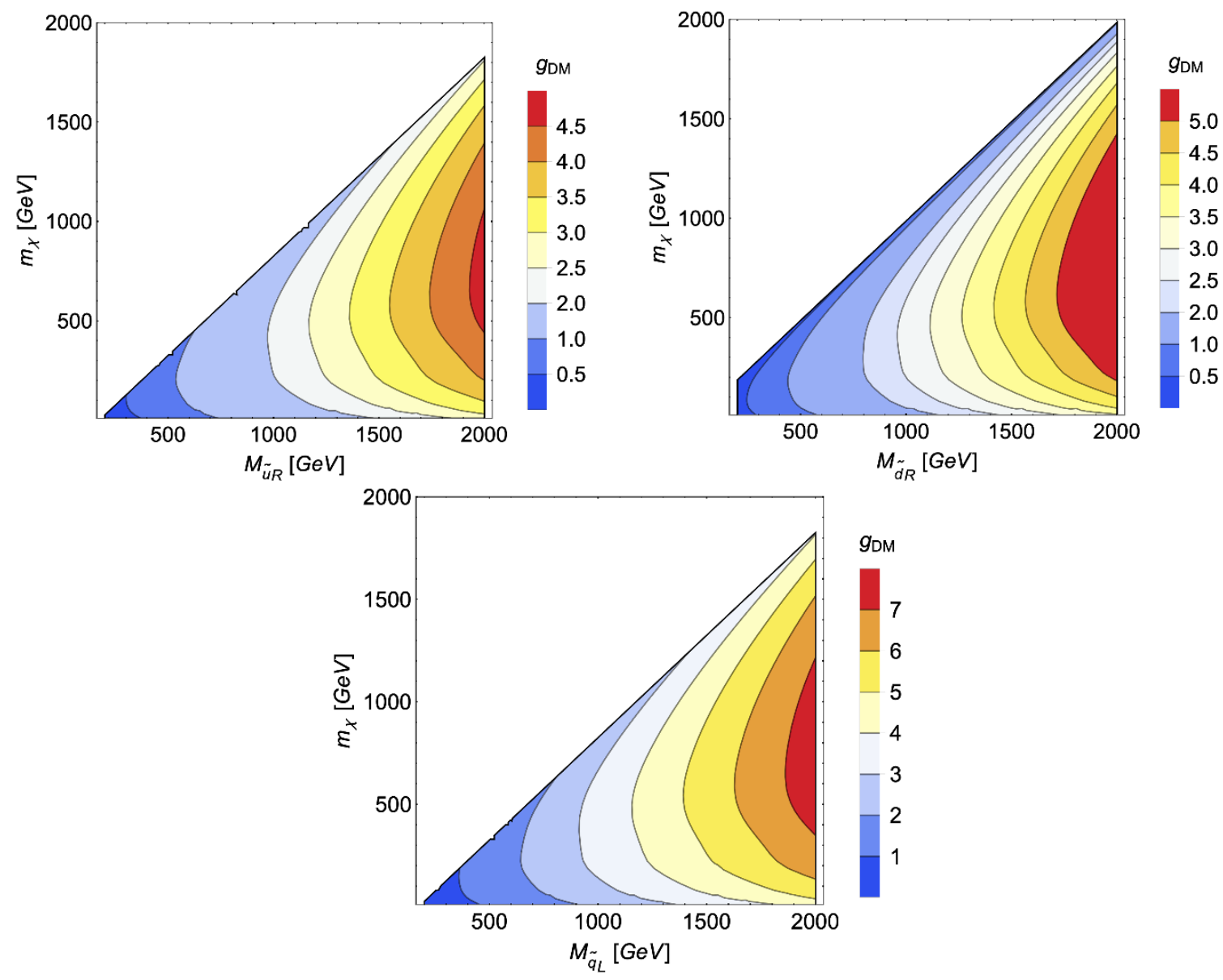

Figure 5. Limits on $g_{D M}$ in the plane of the dark matter and mediator masses from the SD cross section for the $u_{R}$ (upper left), $d_{R}$ (upper right) and $q_{L}$ (bottom) models.

the null searches of the Xenon $1 \mathrm{~T}$ experiment [31]. In figure 6 we show limits on $g_{D M}$ for the $\left(q_{L}, u_{R}, d_{R}\right)$ models in the plane of the dark matter and mediator masses. The shaded regions correspond to allowed values of $g_{D M}$. Comparing with figure 5 , we observe that despite being suppressed because they arise at higher orders, the SI limits are stronger (typically by about an order of magnitude) than the SD limits in the entire parameter space, and become very strong $\left(g_{D M} \lesssim 0.1\right)$ in the resonant region.

\section{Dark matter production at the LHC}

At the LHC, there are three short distance processes of interest, as predicted by this model, which lead to missing transverse momentum $\left(\mathbb{E}_{T}\right)$ signatures:

- pair production of the colored mediators $(\tilde{q})$, followed by their decay into dark matter $(\chi)$ plus a quark;

- associated production of $\tilde{q}$ with $\chi$; and 

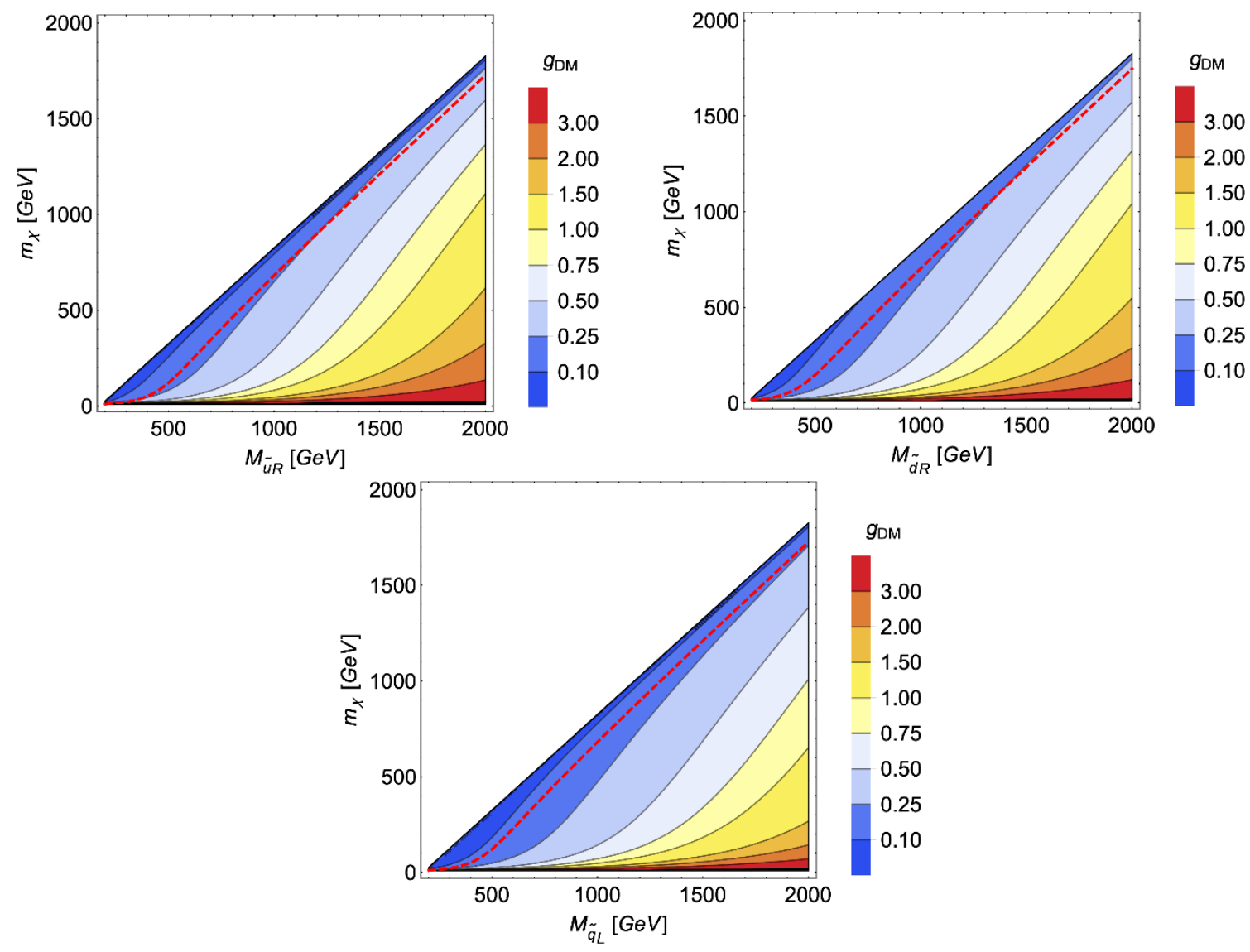

Figure 6. Limits on $g_{D M}$ in the plane of the dark matter and mediator masses from the SI cross section for the $u_{R}$ (upper left), $d_{R}$ (upper right) and $q_{L}$ (bottom) models. The red dashed line indicates the region of parameter space above which the partial width for the mediators is less than $\Lambda_{Q C D}$.
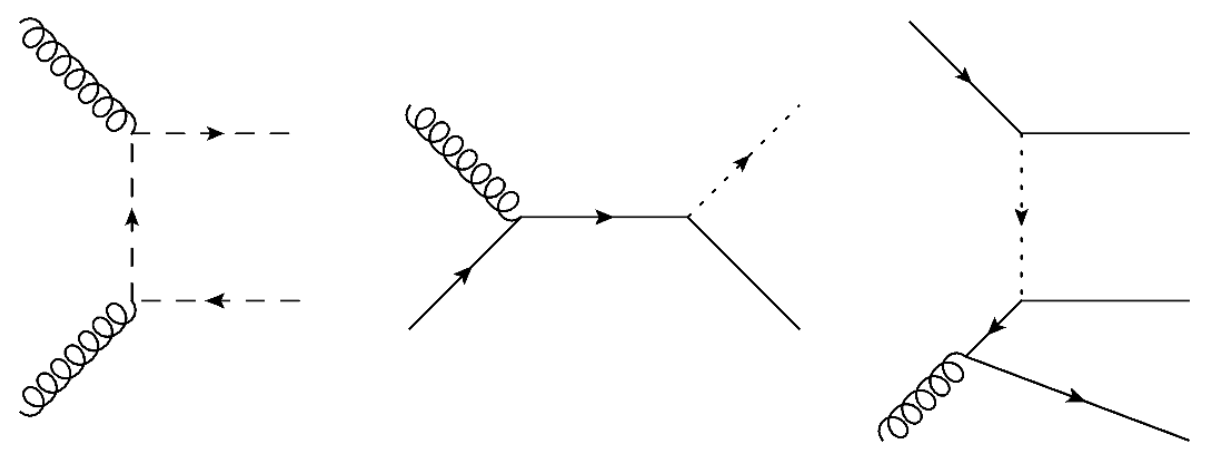

Figure 7. Representative Feynman diagrams for (left to right): mediator pair production, associated production, and $\chi \chi+$ jet production at the LHC. 

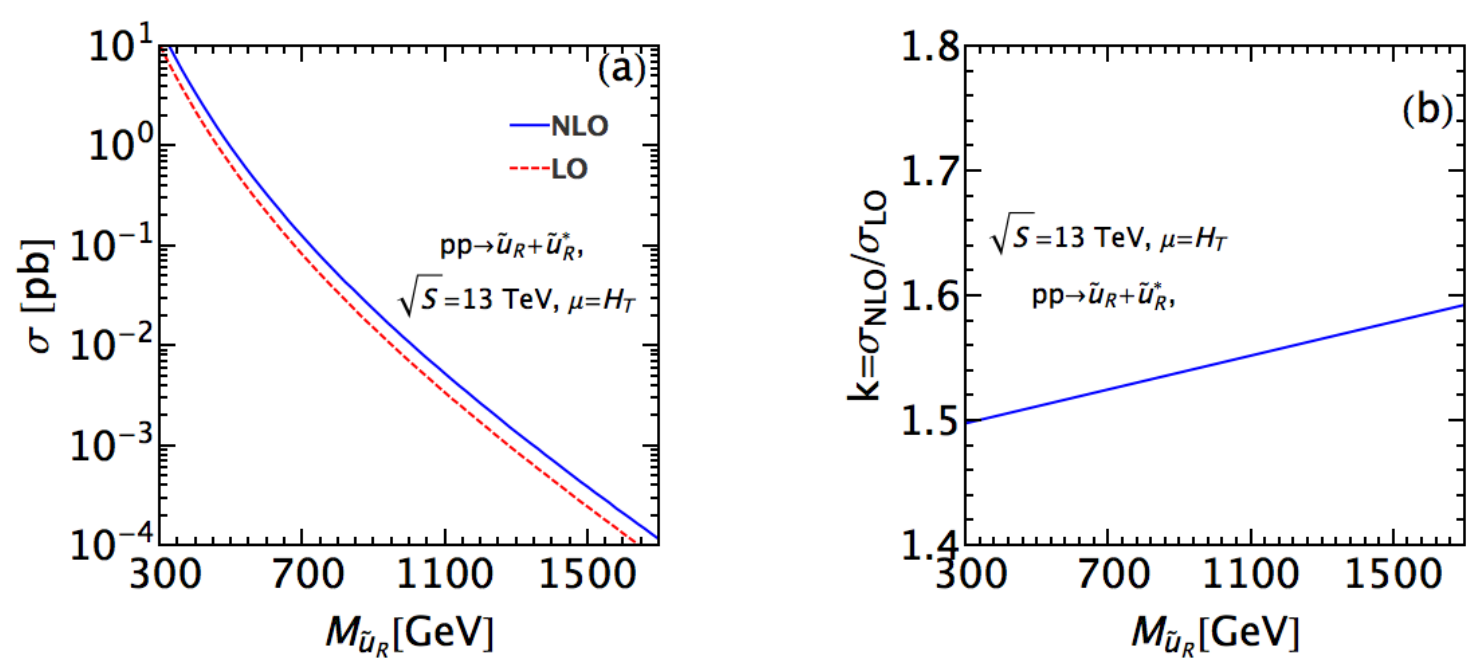

Figure 8. The production cross section (left) and $k$-factor (right) for mediator pair production via QCD at the $13 \mathrm{TeV}$ LHC. Here, we have summed over contributions from the $\tilde{q} \tilde{q}, \tilde{q} \tilde{q}^{*}$ and $\tilde{q}^{*} \tilde{q}^{*}$ final states in each case.

- pair production of the dark matter in association with a jet from initial state radiation, $p p \rightarrow \chi \chi j$.

The first processes are dominated by production of the mediators through the strong force (for $g_{D M} \ll g_{s}$ ) yielding a jets $+\mathbb{E}_{T}$ signature. A special case has a top-flavored mediator, whose decay into a top quark often also results in charged leptons and bottom-flavored quarks in the final state. The rate of the associated production of $\tilde{q}$ and $\chi$ and dark matter pair production processes are controlled by $g_{D M}$. For the parameter space of interest, the dark matter pair production process is always subdominant, and will be neglected from here on.

\subsection{Cross-sections at NLO}

Robust interpretations of LHC data require comparison with precise theoretical determinations of cross sections. We compute the inclusive rates for the mediator pair and associated production processes at NLO in QCD. These calculations are performed in the MG5_aMC@NLO framework [32], with the simplified models implemented via FeynRules [33]. One-loop corrections are computed in MadLoop, based on the OPP method [34], with ultraviolet divergences and rational $R_{2}$ terms via the NLOCT package [35]. CT14NNLO PDF sets are employed in both LO and NLO QCD results [36], with factorization and renormalization scale chosen to be $\mu_{f}=\mu_{r}=\frac{1}{2} H_{T}=\frac{1}{2} \sum_{i} \sqrt{p_{T, i}^{2}+m_{i}^{2}}$, where the sum is over all of the final-state particles.

The amplitude for pair production of mediators from initial state gluons is induced entirely from QCD interactions, whereas the quark-initiated subprocess receives both a QCD contribution and one of order $g_{D M}^{2}$ from dark matter exchange. The pure QCD contribution results in a $\tilde{q} \tilde{q}^{*}$ final state, and the only unknown parameter entering into the 

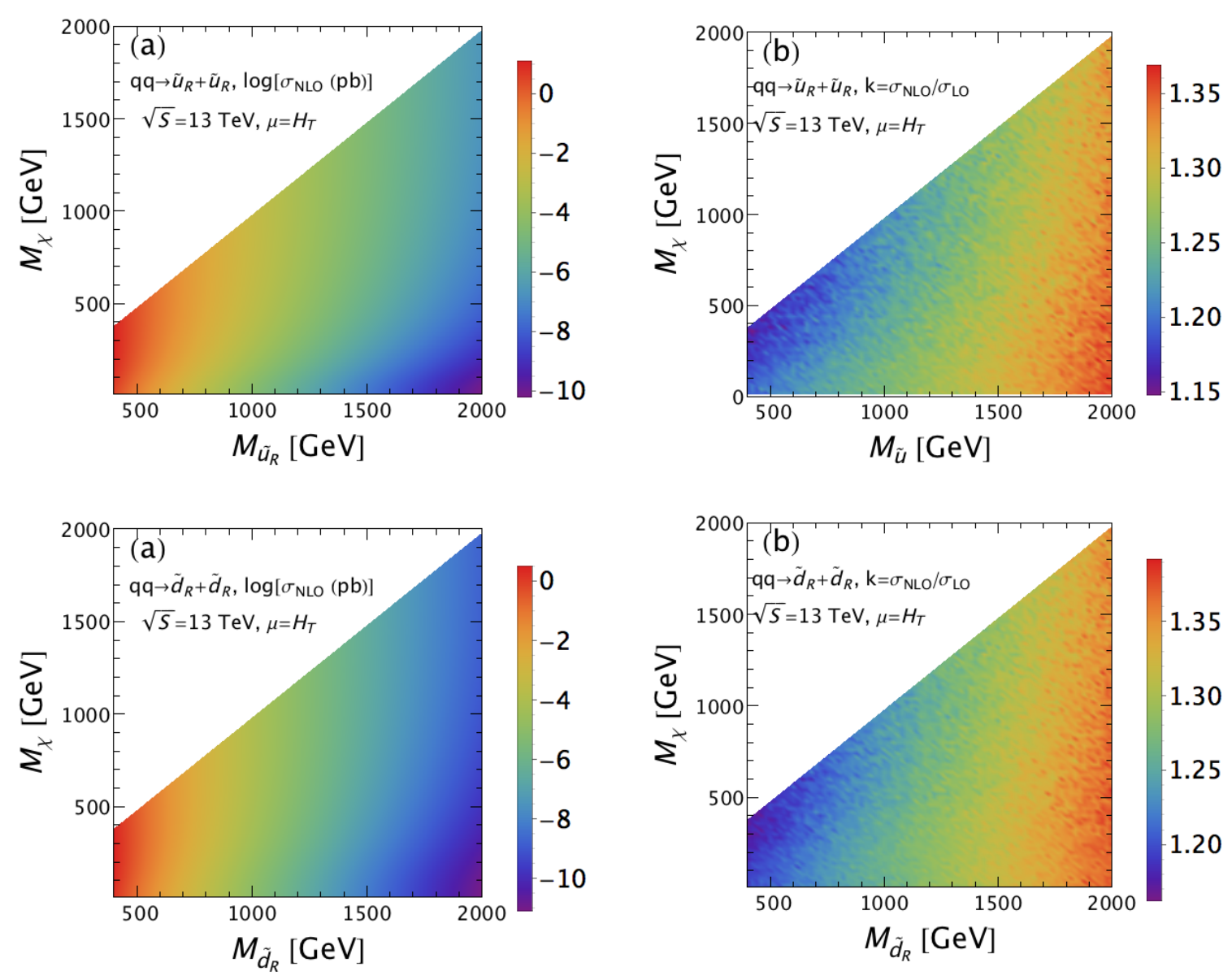

Figure 9. The production cross section and $k$-factor for mediator pair production via dark matter exchange in the $u_{R}$ model (upper) and $d_{R}$ model (lower) at the $13 \mathrm{TeV}$ LHC.

rate is the mediator mass itself. In figure 8, we show the LO and NLO QCD cross sections at the $\sqrt{s}=13 \mathrm{TeV}$ LHC as a function of the mediator mass, as well as the $k$-factor, defined as $k \equiv \sigma_{N L O} / \sigma_{L O}$. At this order, the production cross section for the $q_{L}$ model is the sum of the results for the $u_{R}$ and $d_{R}$ models. Evident from the figure, the $k$-factor is fairly flat in the mediator mass, with a value of about $\sim 1.5$ to 1.6.

The dark matter exchange contributions produce (for Majorana $\chi$ ) $\tilde{q} \tilde{q}^{*}, \tilde{q} \tilde{q}$, and $\tilde{q}^{*} \tilde{q}^{*}$ final states, with rates which strongly depend on both $g_{D M}$ and $m_{\chi}$. Since the scaling $\propto g_{D M}^{4}$ is simple, we set $g_{D M}=1$ as our benchmark point. The production cross section and $k$ factor in the $u_{R}$ and $d_{R}$ models are shown in the figure 9 , summing over the $\tilde{q} \tilde{q}^{*}$, $\tilde{q} \tilde{q}$, and $\tilde{q}^{*} \tilde{q}^{*}$ final states in each case. While the cross sections fall steeply as the mediator mass increases, they increase with larger $m_{\chi}$, as can be understood by the fact that the $\tilde{q} \tilde{q}$ and $\tilde{q}^{*} \tilde{q}^{*}$ final states violate fermion number, and thus require an insertion of the Majorana mass to be nonzero.

In figure 10, we show the NLO cross section for associated production of dark matter and a mediator in the $u_{R}$ and $d_{R}$ models. As the $s$-channel Feynman diagram dominates, 

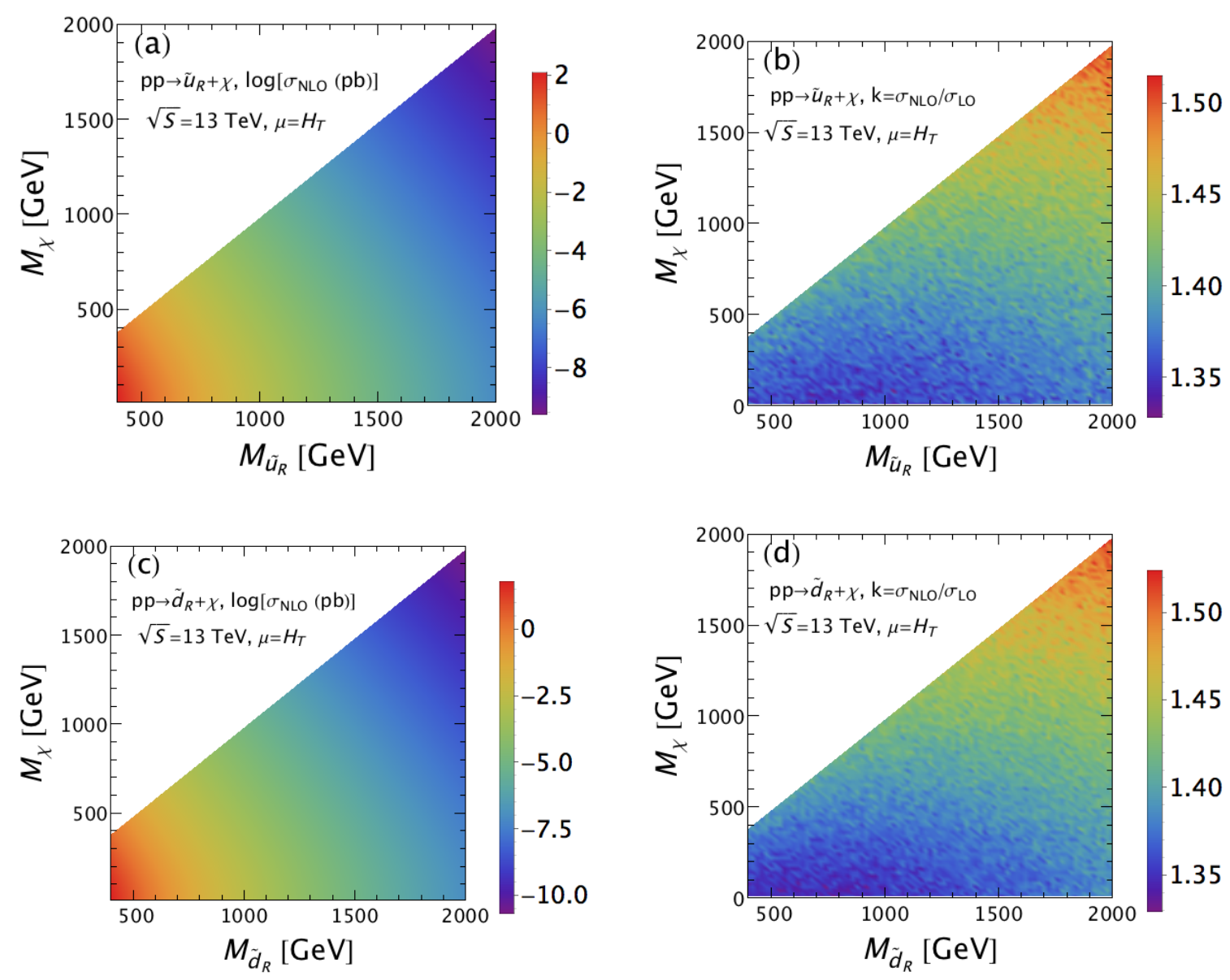

Figure 10. The production cross section and $k$-factor for associated mediator plus dark matter production in the $u_{R}$ model (upper) and $d_{R}$ model (lower) at the $13 \mathrm{TeV}$ LHC.

the cross-section falls rapidly with increasing mediator plus dark matter mass. However, we find that the $k$ factor increases with larger invariant mass from $\sim 1.35$ to $\sim 1.5$.

\subsection{Experimental searches}

We assess the existing constraints from the null results of LHC searches for signatures including missing transverse momentum by implementing two representative searches based on $\sim 36 \mathrm{fb}^{-1}$ of integrated luminosity collected during operation at $\sqrt{s}=13 \mathrm{TeV}$ :

- the mono-jet $+\mathbb{E}_{T}$ search by ATLAS [37]; and

- the multi-jets $+\mathbb{E}_{T}$ search by CMS [38].

In each case, we implement the search in the framework of Madanalysis5 [39], validate it against the experimental result, and re-cast limits on the simplified model parameter space.

\subsubsection{Mono-jet $+\boldsymbol{E}_{T}$ search}

The ATLAS family of mono-jet $+\mathbb{E}_{T}$ search [37] selects events with: 
- a leading anti- $k_{T}$ jet with $p_{T}^{j} \geq 250 \mathrm{GeV}$ in the central region, $|\eta|<2.4$;

- missing transverse momentum $\mathbb{E}_{T} \geq 250 \mathrm{GeV}$;

- up to four sub-leading anti- $k_{T}$ jets with $p_{T}>30 \mathrm{GeV}$ and $|\eta|<2.8$;

- $\Delta \phi\left(\right.$ jet, $\left.\mathbb{E}_{T}\right)>0.4$ between the missing transverse momentum and any jet;

- no isolated electrons (muons) with $p_{T}>20(10) \mathrm{GeV}$.

Events passing the selection cuts are further sorted into a variety of inclusive and exclusive bins in $\mathbb{E}_{T}$. Despite the moniker "mono-jet", this search does not veto sub-leading jets, and is thus sensitive to $\mathbb{E}_{T}$ plus one, two, or three jet topologies.

The analysis is implemented within the MadAnalysis5 framework, with the recasted code, as well as the details of validation are documented in the MadAnalysis5 database [40]. We follow the expirmental paper in order to recast the analysis. Anti- $k_{T}$ jets reconstructed using FAST JET [41], with a jet radius of 0.4, and the detector simulated by Delphes3 [42], with parameters tuned to match the ATLAS specifications. Since the detailed cut flows were not available for this analysis, it is validated by reproducing the exclusion curve for the supersymmetric process of scalar top production with decay $\tilde{t}_{1} \rightarrow c \chi_{1}^{0}$ [37]. The validated analysis is found to reproduce the experimental benchmark very closely.

Signal events for mediator pair and associated production are generated at tree level in MadGraph5 with up to two additional jets, and subsequently showered and hadronized using PYTHIA8 [43], with matrix element and parton shower (ME-PS) merging [44], performed using a merging parameter of $m_{\tilde{q}} / 4$. The rates are normalized to the NLO cross sections discussed above. Based on the normalized number of events obtained for each signal region, we apply the $\log$ likelihood method to obtain an exclusion at each point in the $m_{\tilde{q}}-m_{\tilde{\chi}}$ plane. MadAnalysis5 is used to handle the event selection and to compute the associated upper limit at the $95 \%$ confidence level (CL) on the signal cross section according to the CLs technique. Although the analysis contains a large number of signal regions, the upper bound on the cross section at each point is determined predominantly from signal regions that have large signal rates, low background rates and small uncertainties. ${ }^{4}$

In figure 11 we show the resulting constraints on the $u_{R}, d_{R}$ and $q_{L}$ simplified models from analyzing the associated production process. For most of the parameter space, the constraints are fairly weak, with the strongest constraints applying to the $q_{L}$ model. Figure 12 shows constraints from mediator pair production. While they are typically stronger than the associated production constraints, they are also rather weak for constraining most of the parameter space. However, for the lower range of masses considered, there is a region where QCD production of the mediators saturates the limit, implying that these regions are decisively ruled out for any value of $g_{D M}$, resulting in a prompt mediator decay. We also note that as one approaches the degenerate limit, $M_{\tilde{q}} \sim m_{\chi}$, the bounds from pair production rapidly become ineffective as the jets from mediator decay become soft. In addition, constraints get weaker for smaller $m_{\chi}$, which suppresses the $\tilde{q} \tilde{q}$ and $\tilde{q}^{*} \tilde{q}^{*}$ subprocesses (this

\footnotetext{
${ }^{4}$ The bulk of the exclusion originates from signal regions IM8-IM10 and EM8-EM10, the high $p_{T}$ and $\mathbb{E}_{T}$ regions as described in [37].
} 

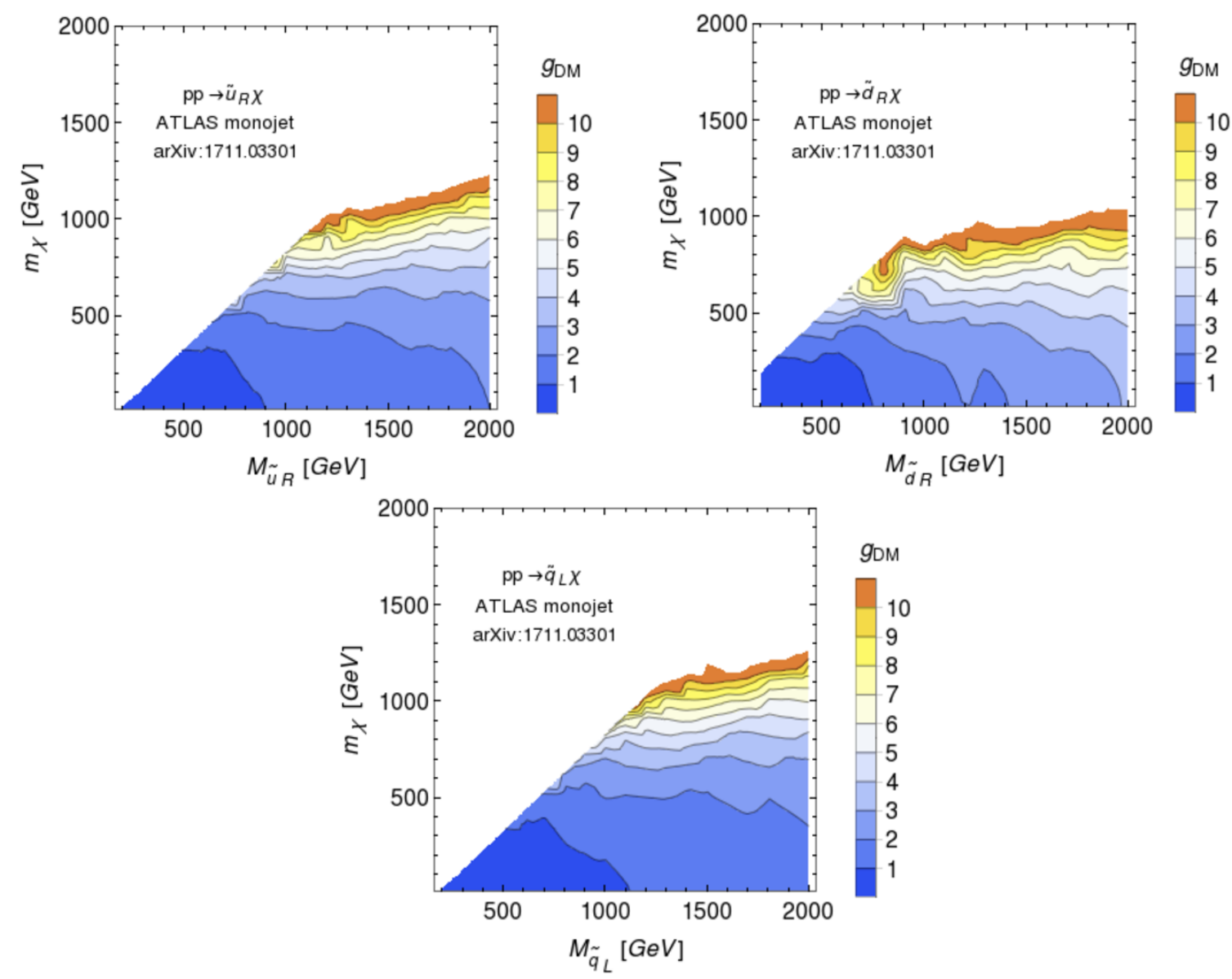

Figure 11. ATLAS mono-jet constraints on $g_{D M}$ derived from associated production for the $u_{R}$ (upper left), $d_{R}$ (upper right) and $q_{L}$ (lower) models. Despite the moniker "mono-jet", this search does not veto sub-leading jets, and is thus sensitive to $\mathbb{E}_{T}$ plus one, two, or three jet topologies.

is particularly pronounced for larger mediator masses for which the $g g$ parton flux is less important relative to $q q$ ). In contrast, the associated production constraints of figure 11 has a much flatter behavior, with constraints simply getting stronger with falling $m_{\chi}$.

\subsubsection{Multi-jet $+\boldsymbol{E}_{T}$ search}

The other LHC search of interest is the CMS search in the 0 lepton + jets $+\mathbb{E}_{T}$ channel [38] with an integrated luminosity of $36.2 \mathrm{fb}^{-1}$. It is designed to search for colored supersymmetric particles, gluinos and squarks, decaying as: $\tilde{g} \rightarrow q \bar{q} \chi_{1}^{0}, \tilde{q} \rightarrow q \chi_{1}^{0}$ and $\tilde{t}_{1} \rightarrow t \chi_{1}^{0}$. A first set of baseline selections are applied as follows: $N_{j} \geq 2, H_{T} \geq 300 \mathrm{GeV}$, MHT > $300 \mathrm{GeV}$, and $\Delta(\phi, j)>0.5$. Eventually, events are sorted into multiple signal regions in bins of the number of jets $\left(n_{j}\right)$, the scalar sum of all jet transverse momenta $\left(H_{T}\right)$, and the vector sum of all jet $p_{T}(\mathrm{MHT})$. For each bin of $n_{j}$, there are 10 different signal regions in bins of $H_{T}$ and MHT in intervals of $50 \mathrm{GeV}, 150 \mathrm{GeV}$ and $250 \mathrm{GeV}$. To facilitate validation, in addition to the exclusion curves for specific channels, representative cutflows for a few benchmark points are provided. The analysis been recast within the framework 

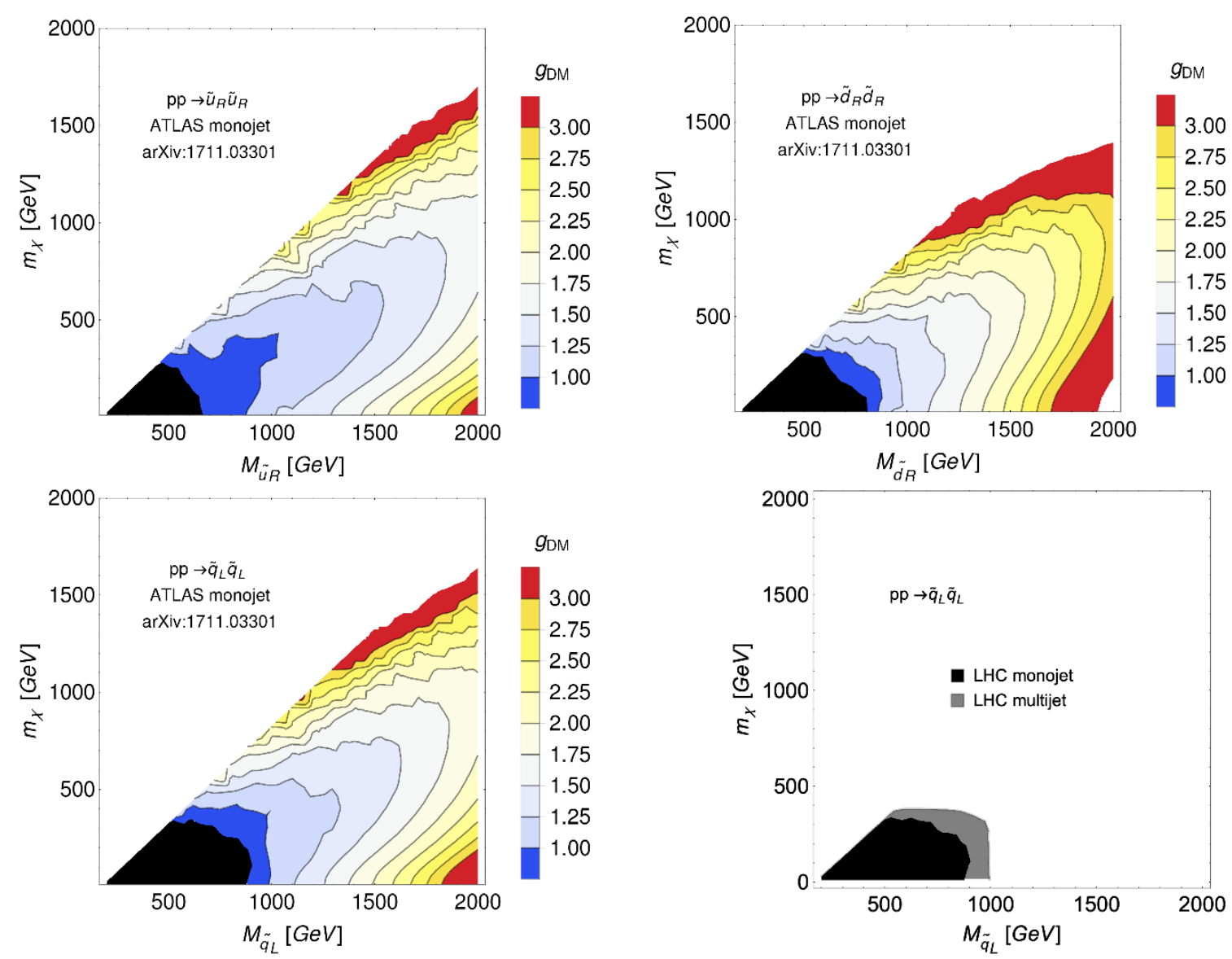

Figure 12. ATLAS monojet constraints on $g_{D M}$ derived from mediator pair production for the $u_{R}$ (upper left), $d_{R}$ (upper right) and $q_{L}$ (lower left) models. Despite the moniker "mono-jet", this search does not veto sub-leading jets, and is thus sensitive to $\mathbb{E}_{T}$ plus one, two, or three jet topologies. In the lower right panel, LHC monojet and multijet constraints for the $q_{L}$ model are compared. The black and grey regions correspond to exclusion independent of the value of $g_{D M}$ for the two different experimental analyses, as indicated.

of MadAnalysis5 [45], where it is found that the recast cutflows typically agree with the experimental ones to within $10 \%$.

Proceeding as before, constraints on the production of dark matter and/or mediators are derived from signal events produced with MadGraph5. The most important process is found to be mediator pair production, and constraints once again lead to a region which is ruled out for any value of $g_{D M}$, resulting in a prompt mediator decay, because QCD production saturates the limits. In the lower left panel of figure 12, we compare the excluded region from the mono-jet and multi-jet searches. The multi-jet search has constrained a somewhat larger region in the plane of the mediator and dark matter masses. It should be noted that one can also obtain the limits on $g_{D M}$ from this channel given that the limits are a bit more constraining than the monojet channel. However, since the direct detection constraints are significantly more constraining, we shall not perform that exercise here. 

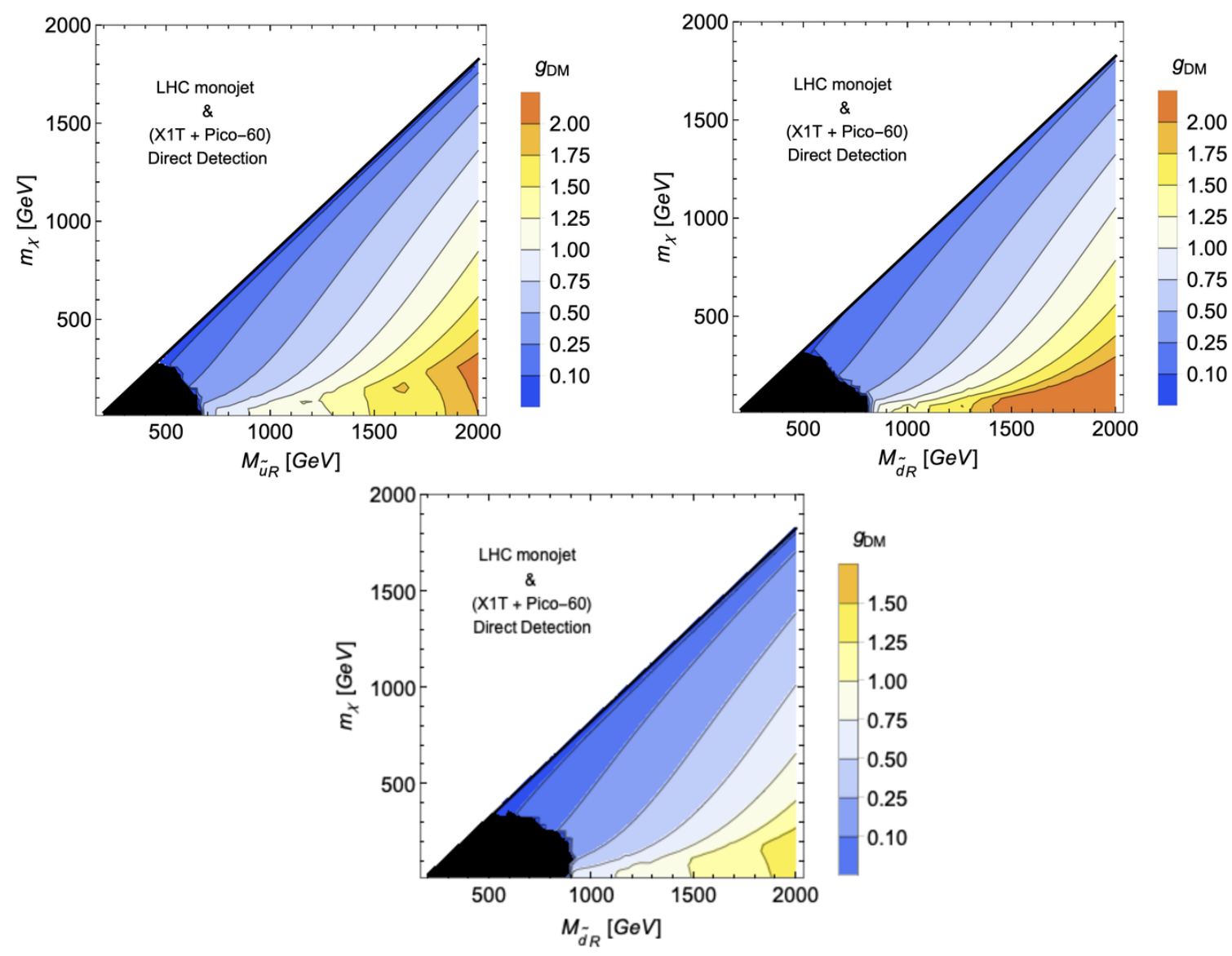

Figure 13. Combined SI, SD and LHC constraints for the $u_{R}$ (upper left), $d_{R}$ (upper right) and $q_{L}$ (lower) models. Shaded regions indicate allowed values of the coupling $g_{D M}$.

\section{Combined limits}

We assemble the combined limits from direct detection and collider searches in figure 13. The black shaded regions are ruled out for any value of $g_{D M}$ leading to prompt mediator decays, by constraints from the LHC, whereas colored shading indicates the maximum allowed $g_{D M}$ at that point. The picture that emerges is that collider and direct searches are highly complementary, with the collider able to rule out regions of parameter space categorically, whereas the direct searches sensitive to SI scattering typically provide the strongest constraints on $g_{D M}$ in the remainder of the parameter space (with colliders filling the region of very tiny dark matter masses). Despite being less suppressed at tree level, the SD constraints are only relevant at large mediator mass $(\sim 2 \mathrm{TeV})$ and small dark matter mass $(\sim 10 \mathrm{GeV})$ and is subdominant for all other regions of parameter space. This highlights the importance that higher order contributions to the SI cross section has on this particular simplified model.

Finally, it is interesting to use the current constraints from colliders and direct searches to construct the largest allowed forecast for the annihilation cross section. At very low velocities $\left(\beta \sim 10^{-3}\right)$, this cross section is probed by indirect searches for high energy 
gamma rays, cosmic rays, or neutrinos produced by dark matter annihilation in the Galaxy. In the early Universe, under the assumption that the extrapolation to the time of freeze-out follows a standard cosmology, the inclusive annihilation cross section (for $\beta \sim 1 / 20$ ) maps onto the expected dark matter abundance. In figure 14 we present the velocity averaged annihilation cross-section in the non relativistic limit. We import the model files written in Feynrules in micrOMEGAs5.0 [46] to evaluate $\left\langle\sigma v_{a n n}\right\rangle$ for $g_{D M}$ set to its maximally allowed value obtained from figure 13. The black shaded area of figure 14 represents the region of parameter space ruled out by LHC constraints, and the colored shaded regions correspond to different values of $\langle\sigma v\rangle$ normalized to $10^{-26} \mathrm{~cm}^{3} / \mathrm{s} .{ }^{5}$ Both the $q_{L}$ and $u_{R}$ models have larger values of $\langle\sigma v\rangle$ compared to the $d_{R}$ model. This can be understood from the velocityaveraged annihilation cross-section into SM fermions,

$$
\langle\sigma v\rangle \simeq N_{c}^{f} g_{D M}^{4}\left[\frac{m_{f}^{2} \sqrt{1-\frac{m_{f}^{2}}{m_{\chi}^{2}}}}{64 \pi\left(m_{\tilde{q}}^{2}+m_{\chi}^{2}-m_{f}^{2}\right)^{2}}+\beta^{2}\left\{\frac{m_{\chi}^{2} \sqrt{m_{\chi}^{4}+m_{\tilde{q}}^{4}}}{32 \pi\left(m_{\chi}^{2}+m_{\tilde{q}}^{2}\right)^{4}}+\mathcal{O}\left(m_{f}^{2}\right)\right\}\right],
$$

where $N_{c}^{f}$ is the appropriate color factor for the species of fermion $f$, and $\beta$ is the velocity of the colliding DM particles (Mandelstam $s=4 m_{\chi}^{2} /\left(1-\beta^{2}\right)$ ), which is about $\sim 10^{-3}$. The first term is the velocity independent ( $s$ wave scattering) part of the cross section, while the second piece is the velocity dependent part of the annihilation ( $p$ wave scattering). For simplicity, in the term proportional to $\beta^{2}$, we only show the part of the expression that is independent of the quark mass $\left(m_{f}\right)$. The cross-section at zero velocity is proportional to the square of the quark mass, and in the $q_{L}$ and $u_{R}$ models is dominated by annihilation into top quarks when kinematically accessible. Annihilation to light quarks is dominated by the $p$-wave contribution which is proportional to $\beta^{2}$ and is therefore suppressed. This is also the reason why $\langle\sigma v\rangle$ has a sharp increases for the $q_{L}$ and $u_{R}$ models at the top threshold where the $s$-wave dominates the contribution.

\section{Outlook}

The identity of the dark matter remains one of the most pressing questions confronting particle physics, and the wealth of information from colliders, searches for scattering with nuclei, and searches for dark matter annihilation complement each other in terms of making progress toward that goal. As the precision of the experimental searches increases, there is a need for a corresponding improvement in theoretical predictions, in order to realize the full potential of the experimental data.

We have considered the class of $t$-channel models, in which the dark matter is a Majorana fermion which interacts with quarks at tree level via exchange of colored scalar mediators. At leading order, this model predicts no spin independent scattering with nuclei, suggesting that searches for dark matter scattering with nuclei have much reduced power to constrain it. However, at higher order there are contributions to the spin independent

\footnotetext{
${ }^{5} \mathrm{~A}$ ballpark number for $\langle\sigma v\rangle$ to saturate the DM relic density is $3 \times 10^{-26} \mathrm{~cm}^{3} / \mathrm{s}$, with smaller values indicating overabundant DM for a standard cosmological history.
} 

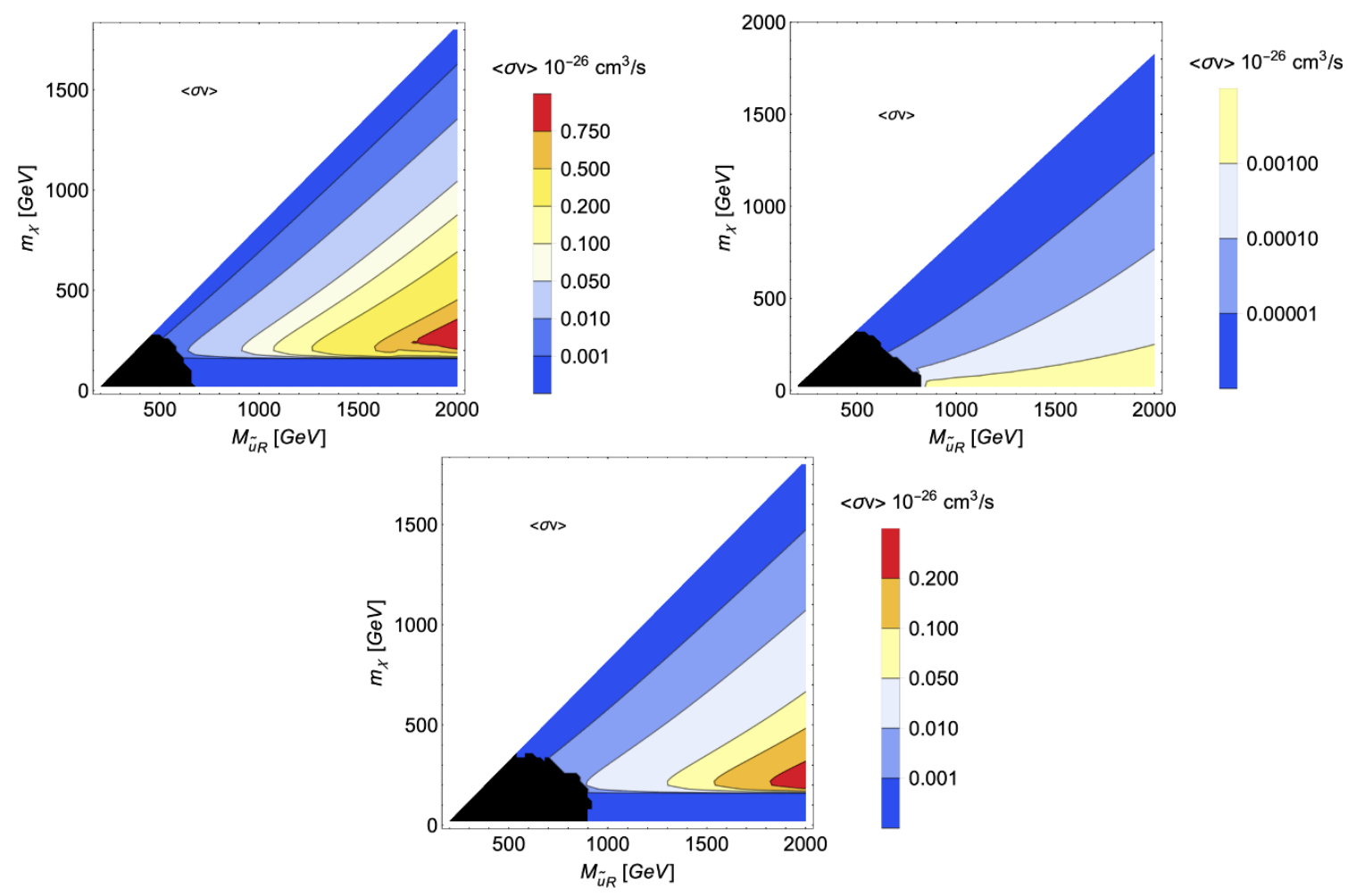

Figure 14. Velocity averaged annihilation cross-sections $(\langle\sigma v\rangle)$ for the $u_{R}$ (upper left), $d_{R}$ (upper right) and $q_{L}$ (lower) models in the zero temperature limit.

cross section, which despite being suppressed, as compared to tree level SD cross-section, are far more constraining because of the coherent enhancement of the experimental limits compared to spin dependent searches. We have assembled a consistent treatment of the Wilson coefficients leading to SI scattering, including one loop contributions to the dark matter coupling to gluons and renormalization group resummation of large logs. Our results highlight the need for higher orders in analysis of the constraints on the parameter space of dark matter models.

Similarly, LHC searches provide key information which allows combinations of masses to be excluded for any value of the dark matter interaction strength. Understanding the reach of these searches accurately requires higher order calculations of the dark matter production processes. We have computed the rates for mediator pair and dark matter plus mediator associated production at next-to-leading order, and find that they significantly improve the bounds extracted from LHC searches compared to the leading order results. While the efficiencies for the searches do not change, the evaluation of NLO cross sections improve the limits by about $75-100 \mathrm{GeV}$.

There are a few directions which are beyond the scope of this work, but would be worthwhile to explore. We have largely neglected the contributions from the top-flavored mediators, because their decays typically produce (on-shell or off-shell) top quarks, whose decays themselves offer a wider range of final states, including high energy charged leptons. There are also regions of parameter space for which the mediator becomes long lived, with 
different (and powerful) constraints coming into play. We look forward to incorporating such refinements in the future. Finally, we would like to direct the interested reader, and in particular our experimental colleagues to the website of this project [47], where the analysis codes and additional plots can be found.

\section{Acknowledgments}

The authors are grateful for conversations with Bakul Agarwal, R. Sekhar Chivukula, Tongyan Lin and Hua-sheng Shao. This work is supported in part by National Science Foundation Grants No. PHY-1519045, PHY-1620638 and PHY-1719914. C.-P. Yuan is also grateful for the support from the Wu-Ki Tung endowed chair in particle physics. This work was supported in part by Michigan State University through computational resources provided by the Institute for Cyber-Enabled Research

\section{A Wilson coefficients}

\section{A.1 Quarkionic Wilson coefficients}

Representative Feynman diagrams for the LO contributions to the processes $q \chi \rightarrow q \chi$ are shown in figure 1 , leading to amplitude

$$
\begin{aligned}
\mathcal{M} & =\left(-i g_{D M}\right)^{2}\left(\bar{\chi} P_{R} u\right) \frac{i}{p^{2}-M_{\tilde{u}}^{2}}\left(\bar{u} P_{L} \chi\right) \\
& \approx\left(\frac{i g_{D M}^{2}}{M_{\tilde{u}}^{2}-m_{\chi}^{2}}+2 \frac{k_{q \nu} k_{\chi}^{\nu}}{\left(M_{\tilde{u}}^{2}-m_{\chi}^{2}\right)^{2}}\right) \frac{1}{8}\left[\left(\bar{\chi} \gamma^{\mu} \chi\right)\left(\bar{u} \gamma_{\mu} u\right)-\left(\bar{\chi} \gamma^{\mu} \gamma^{5} \chi\right)\left(\bar{u} \gamma_{\mu} \gamma_{5} u\right)\right] .
\end{aligned}
$$

Here $k_{q}$ and $k_{\chi}$ are the four momenta of the quark and dark matter respectively. Terms that are order $\left(M_{\tilde{u}}^{2}-m_{\chi}^{2}\right)^{2}$ in the expansion above have explicit momentum factors in the numerator. This matches to the matrix elements generated from the effective lagrangian in equation (3.4) from which we determine the wilson coefficients given in equation (3.11).

\section{A.2 Gluonic Wilson coefficients}

Representative Feynman diagrams for the LO contributions to the process $g \chi \rightarrow g \chi$ are shown in figure 2. Amplitudes are generated with Feynarts [48], decomposed into tensor integrals using FormCalc [49], and the expansion of tensor integrals is performed with Package-X [50]. In order to calculate Wilson coefficients we match amplitudes produced from the full theory in equations (2.3)-(2.5) to that from the EFT of equation (3.4). We calculate $S$-channel production $g\left(k_{1}\right) g\left(k_{2}\right) \rightarrow \chi\left(k_{3}\right) \chi\left(k_{4}\right)$ and apply the following projection 
operators to the amplitude to in order to match amplitudes:

$$
\begin{aligned}
A^{\mu_{1} \mu_{2}}= & i 8 f_{G}\left(k_{2}{ }^{\mu_{1}} k_{1}{ }^{\mu_{2}}-g^{\mu_{1} \mu_{2}}\left(k_{1} \cdot k_{2}\right)\right), \\
B^{\mu_{1} \mu_{2}}=i \frac{g_{G}^{(2)}}{m_{\chi}^{2}} & {\left[g^{\mu_{1} \mu_{2}}\left(2\left(k_{1} \cdot k_{3}\right)\left(k_{2} \cdot k_{3}\right)+2\left(k_{1} \cdot k_{4}\right)\left(k_{2} \cdot k_{4}\right)-\left(k_{3}^{2}+k_{4}^{2}\right)\left(k_{1} \cdot k_{2}\right)\right)\right.} \\
& +k_{1}^{\mu_{2}}\left(\left(k_{3}^{2}+k_{4}^{2}\right) k_{2}^{\mu_{1}}-2\left(k_{3}^{\mu_{1}}\left(k_{2} \cdot k_{3}\right)+k_{4}^{\mu_{1}}\left(k_{2} \cdot k_{4}\right)\right)\right) \\
& +k_{3}^{\mu_{2}}\left(2 k_{3}^{\mu_{1}}\left(k_{1} \cdot k_{2}\right)-2 k_{2}^{\mu_{1}}\left(k_{1} \cdot k_{3}\right)\right) \\
& \left.+2 k_{4}^{\mu_{2}}\left(k_{4}^{\mu_{1}}\left(k_{1} \cdot k_{2}\right)-k_{2}^{\mu_{1}}\left(k_{1} \cdot k_{4}\right)\right)\right]
\end{aligned}
$$

and,

$$
\begin{aligned}
C^{\mu_{1} \mu_{2}}= & i 2 \frac{g_{G}^{(1)}}{m_{\chi}}\left[g^{\mu_{1} \mu_{2}}\left(k_{1} \cdot k_{3}\right) \gamma \cdot k_{2}-g^{\mu_{1} \mu_{2}}\left(k_{1} \cdot k_{4}\right) \gamma \cdot k_{2}+g^{\mu_{1} \mu_{2}}\left(k_{2} \cdot k_{3}\right) \gamma \cdot k_{1}\right. \\
& -g^{\mu_{1} \mu_{2}}\left(k_{2} \cdot k_{4}\right) \gamma \cdot k_{1}+\left(k_{1} \cdot k_{2}\right)\left(g^{\mu_{1} \mu_{2}}\left(\gamma \cdot k_{4}-\gamma \cdot k_{3}\right)+\gamma^{\mu_{2}}\left(k_{3}^{\mu_{1}}-k_{4}^{\mu_{1}}\right)+\gamma^{\mu_{1}}\left(k_{3}^{\mu_{2}}-k_{4}^{\mu_{2}}\right)\right) \\
& -\gamma^{\mu_{2}} k_{2}^{\mu_{1}}\left(k_{1} \cdot k_{3}\right)+\gamma^{\mu_{2}} k_{2}^{\mu_{1}}\left(k_{1} \cdot k_{4}\right)-k_{3}^{\mu_{2}} k_{2}^{\mu_{1}}\left(\gamma \cdot k_{1}\right)+k_{4}^{\mu_{2}} k_{2}^{\mu_{1}} \gamma \cdot k_{1} \\
& \left.+k_{1}^{\mu_{2}}\left(\gamma^{\mu_{1}}\left(k_{2} \cdot k_{4}-k_{2} \cdot k_{3}\right)+k_{2}^{\mu_{1}}\left(\gamma \cdot k_{3}-\gamma \cdot k_{4}\right)+\left(k_{4}^{\mu_{1}}-k_{3}^{\mu_{1}}\right) \gamma \cdot k_{2}\right)\right] .
\end{aligned}
$$

The three projection operators correspond to the three $g g \chi \chi$ vertices orignating from the operators in the effective Lagrangian in equation (3.4).

We define Mandelstam variables as usual:

$$
S=\left(k_{1}+k_{2}\right)^{2}, \quad T=\left(k_{1}-k_{3}\right)^{2}, \quad U=\left(k_{1}-k_{4}\right)^{2},
$$

which satisfy $S+T+U=2 m_{\chi}^{2}$. To simplify the matching we choose a specific phase space point $-U=m_{\chi}^{2}-S / 2$ and $T=m_{\chi}^{2}-S / 2$. The resulting expressions are expanded in powers of $S$ and we extract the Wilson coefficients by matching to the following equations.

$$
\begin{aligned}
& A \cdot(A+B+C)=32 f_{G}^{2} S^{2} \\
& B \cdot(A+B+C)=-2 m_{\chi}^{3} S^{2}\left(m_{\chi} \frac{g_{G}^{(2)}}{m_{\chi}^{2}}+2 \frac{g_{G}^{(1)}}{m_{\chi}}\right)+\mathcal{O}\left(S^{3}\right) \\
& C \cdot(A+B+C)=2 m_{\chi}^{2} S^{2}\left(m_{\chi} \frac{g_{G}^{(2)}}{m_{\chi}^{2}}+4 \frac{g_{G}^{(1)}}{m_{\chi}}\right)+\mathcal{O}\left(S^{3}\right)
\end{aligned}
$$

The Wilson coefficents for the gluonic operators listed in equation (3.4) are found to be,

$$
\begin{aligned}
f_{G}= & \alpha_{s} g_{D M}^{2} m_{\chi}\left[-12 m^{2} M^{4} m_{\chi}^{2}\left(m^{2}-M^{2}+m_{\chi}^{2}\right) \Lambda\left(m_{\chi}^{2} ; m, M\right)\right. \\
& -\left(m-M-m_{\chi}\right)\left(m+M-m_{\chi}\right)\left(m-M+m_{\chi}\right)\left(m+M+m_{\chi}\right) \\
& \left.\times\left\{m^{6}-3 m^{4}\left(2 M^{2}+m_{\chi}^{2}\right)+m^{2}\left(3 M^{4}+2 M^{2} m_{\chi}^{2}+3 m_{\chi}^{4}\right)+\left(M^{2}-m_{\chi}^{2}\right)^{2}\left(2 M^{2}-m_{\chi}^{2}\right)\right\}\right] \\
& \times \frac{1}{192 \pi M^{2}\left(m-M-m_{\chi}\right)^{3}\left(m+M-m_{\chi}\right)^{3}\left(m-M+m_{\chi}\right)^{3}\left(m+M+m_{\chi}\right)^{3}},
\end{aligned}
$$


where

$$
\begin{aligned}
\Lambda\left(m_{\chi}^{2} ; m, M\right) & =\frac{\lambda}{m_{\chi}^{2}} \log \left(\frac{m^{2}+\lambda+M^{2}-m_{\chi}^{2}}{2 m M}\right) \\
\lambda & =\sqrt{m^{4}-2 m^{2} M^{2}-2 m^{2} m_{\chi}^{2}+M^{4}-2 M^{2} m_{\chi}^{2}+m_{\chi}^{4}} .
\end{aligned}
$$

Also,

$$
\begin{aligned}
& \frac{g_{G}^{(2)}}{m_{\chi}^{2}}=\alpha_{s} g_{D M}^{2}\left[2 m _ { \chi } ^ { 2 } \left\{10 m_{\chi}^{4}\left(m^{6}-M^{6}\right)+m_{\chi}^{8}\left(m^{2}-5 M^{2}\right)-5 m_{\chi}^{2}\left(m^{2}-M^{2}\right)^{3}\left(m^{2}+M^{2}\right)\right.\right. \\
& \left.+\left(m^{2}-M^{2}\right)^{5}+2 m_{\chi}^{6}\left(-4 m^{4}+2 m^{2} M^{2}+5 M^{4}\right)+m_{\chi}^{10}\right\} \Lambda\left(m_{\chi}^{2} ; m, M\right) \\
& -\left(m-M-m_{\chi}\right)\left(m+M-m_{\chi}\right)\left(m-M+m_{\chi}\right)\left(m+M+m_{\chi}\right) \\
& \times\left\{7 m_{\chi}^{4}\left(m^{4}-M^{4}\right)-2 m_{\chi}^{6}\left(m^{2}-4 M^{2}\right)-2 m_{\chi}^{2}\left(m^{2}-M^{2}\right)^{3}\right. \\
& \left.\left.+2\left(m^{4}-2 m^{2}\left(M^{2}+m_{\chi}^{2}\right)+\left(M^{2}-m_{\chi}^{2}\right)^{2}\right)^{2} \log \left(\frac{m}{M}\right)-3 m_{\chi}^{8}\right\}\right] \\
& \times \frac{1}{48 \pi m_{\chi}^{5}\left(m-M-m_{\chi}\right)^{3}\left(m+M-m_{\chi}\right)^{3}\left(m-M+m_{\chi}\right)^{3}\left(m+M+m_{\chi}\right)^{3}}, \\
& \frac{g_{G}^{(1)}}{m_{\chi}}=\alpha_{s} g_{D M}^{2}\left[2 m_{\chi}^{2}\left(3 m_{\chi}^{2}\left(M^{4}-m^{4}\right)+m_{\chi}^{4}\left(5 m^{2}+M^{2}\right)+\left(m^{2}-M^{2}\right)^{3}-3 m_{\chi}^{6}\right) \Lambda\left(m_{\chi}^{2} ; m, M\right)\right. \\
& +2\left(m+M-m_{\chi}\right)\left(m-M+m_{\chi}\right)\left(m+M+m_{\chi}\right)\left\{m_{\chi}^{2}\left(m-M-m_{\chi}\right)\left(m^{2}-M^{2}-3 m_{\chi}^{2}\right)\right. \\
& \left.\left.-\left(m+M-m_{\chi}\right)\left(m-M+m_{\chi}\right)\left(-m+M+m_{\chi}\right)^{2}\left(m+M+m_{\chi}\right) \log \left(\frac{m}{M}\right)\right\}\right] \\
& \times \frac{1}{192 \pi m_{\chi}^{4}\left(m+M-m_{\chi}\right)^{2}\left(m-M+m_{\chi}\right)^{2}\left(-m+M+m_{\chi}\right)^{2}\left(m+M+m_{\chi}\right)^{2}} .
\end{aligned}
$$

\section{A.3 Gluonic Wilson coefficients using the Fock-Schwinger gauge}

The determination of gluonic Wilson Coefficients can be greatly simplified by using the Fock-Schwinger gauge (for details, see for e.g., References [51] and [22] and references therein), for which

$$
x^{\mu} A_{\mu}^{a}(x)=0 .
$$

In this gauge one can express the gluon field in terms of its field strength tensor $G^{\mu \nu}$, maintaining explicit gauge invariance at each step. The Wilson coefficients can be extracted from the one loop contribution to a Majorana fermion propagating through a non-zero background of gluon fields. The gluonic background modifies the form of the internal quark and mediator propagators. Calculations are performed using FeynCalc-9.20 [52], and we summarize the results corresponding to a single flavor of quark here.

$$
f_{G}=\frac{\alpha_{s}}{4 \pi} \frac{1}{8}\left(\int \frac{d^{4} q}{i \pi^{2}} \frac{\not p+\not q}{\left((p+q)^{2}-m_{q}^{2}\right)^{4}\left(q^{2}-M_{\tilde{q}}^{2}\right)}+\int \frac{d^{4} q}{i \pi^{2}} \frac{\not p+\not q}{\left((p+q)^{2}-m_{q}^{2}\right)\left(q^{2}-M_{\tilde{q}}^{2}\right)^{4}}\right) .
$$

These integrals maybe solved by introducing Feynman parameters to yield the result in equation (A.7). 
For the twist-2 operators we can write out the scattering amplitude as follows and then use projection operators to match and find the Wilson coefficients.

$$
\begin{aligned}
\mathcal{M}_{t 2}= & -\frac{\alpha_{s}}{4 \pi} \bar{\chi} \int \frac{d^{4} q}{i \pi^{2}} \frac{1}{8} \frac{(\not p+\not q)\left(\left(3 m_{\tilde{q}}^{2}-q^{2}\right) g^{\mu \nu}-4 q^{\mu} q^{\nu}\right)}{\left((p+q)^{2}-m_{q}^{2}\right)^{4}\left(q^{2}-m_{\tilde{q}}^{2}\right)} \chi G_{\mu}^{a \rho} G_{\rho \nu}^{a} \\
& -\frac{\alpha_{s}}{4 \pi} \bar{\chi} \int \frac{d^{4} q}{i \pi^{2}} \frac{1}{4} \frac{(\not p+\not q)\left(\left(m_{q}^{2}-2(p+q)^{\mu}(p+q)^{\nu}\right)\right.}{\left((p+q)^{2}-m_{q}^{2}\right)\left(q^{2}-m_{\tilde{q}}^{2}\right)^{4}} \chi G_{\mu}^{a \rho} G_{\rho \nu}^{a} \\
& +\frac{\alpha_{s}}{4 \pi} \bar{\chi} \int \frac{d^{4} q}{i \pi^{2}} \frac{1}{4} \frac{\left(\gamma^{\nu}(p+q)^{\mu}+\gamma^{\mu}(p+q)^{\nu}\right)}{\left((p+q)^{2}-m_{q}^{2}\right)\left(q^{2}-m_{\tilde{q}}^{2}\right)^{3}} \chi G_{\mu}^{a \rho} G_{\rho \nu}^{a},
\end{aligned}
$$

The integrals above can be solved by introducting Feynman parameters and the final result is given in equations (A.9) and (A.10).

\section{B Numerical values}

We list here the various values that we have used in our numerical analysis. Light quark masses are taken from PDG [53] and are defined in the $\overline{\mathrm{MS}}$ scheme at $\mu=2 \mathrm{GeV}$.

$$
\begin{aligned}
& m_{u}=2.2 \mathrm{MeV} \text {, } \\
& m_{d}=4.7 \mathrm{MeV}, \\
& m_{s}=95 \mathrm{MeV} \text {, } \\
& m_{c}=1.3 \mathrm{GeV} \text {, } \\
& m_{b}=4.2 \mathrm{GeV} \text {, } \\
& m_{t}=172 \mathrm{GeV} \text {, } \\
& m_{Z}=91.188 \mathrm{GeV} \text {, } \\
& \alpha_{s}\left(m_{Z}\right)=0.1184 \text {, } \\
& m_{n}=0.9396 \mathrm{GeV} \\
& m_{p}=0.9383 \mathrm{GeV} \text {. }
\end{aligned}
$$

Values of hadronic matrix elements for the spin-0 operators evaluated at the scale $\mu=2 \mathrm{GeV}$ are taken from reference [26] and are given below.

$$
\begin{aligned}
& {\left[f_{T_{u}}\right]_{p}=0.018,} \\
& {\left[f_{T_{d}}\right]_{p}=0.030,} \\
& {\left[f_{T_{s}}\right]_{p}=0.043,} \\
& {\left[f_{T_{u}}\right]_{n}=0.015,} \\
& {\left[f_{T_{d}}\right]_{n}=0.034 \text {, }} \\
& {\left[f_{T_{s}}\right]_{n}=0.043 \text {, }} \\
& \left.f_{T_{G}}\right|_{\mathrm{NNNLO}}=0.80 .
\end{aligned}
$$

Here $\left[f_{T_{x}}\right]_{y}$ corresponds to the contribution of the $x$ quark to the nucleon matrix elements for the nucleon $y \cdot f_{T_{G}}$ is determined using the sum rule

$$
f_{T_{G}}=-\frac{9 \alpha_{S}(\mu)}{4 \pi \beta(\mu)}\left[1-\left(1+\gamma_{m}(\mu)\right) \sum_{u, d, s} f_{T q}\right] .
$$

Here $\beta(\mu)$ and $\gamma_{m}(\mu)$ are the QCD beta function and quark anomalous dimension respectively. Here we calculate $f_{T_{G}}$ by using expressions of $\beta(\mu)$ and $\gamma_{m}(\mu)$ up to order $\mathrm{N}^{3} \mathrm{LO}$ in $\alpha_{s}$. For details on how to calculate $f_{T_{G}}$, see for example, Reference [26]. Hadronic matrix elements for twist-2 operators defined in equation (3.4), also defined at the scale $\mu=2 \mathrm{GeV}$, are extracted from the CT14NNLO parton distribution functions [36].

$$
\begin{array}{rlrl}
{[u(2)+\bar{u}(2)]_{p}} & =0.3481, & {[d(2)+\bar{d}(2)]_{p}} & =0.1902, \\
{[s(2)+\bar{s}(2)]_{p}} & =0.0352, & {[c(2)+\bar{c}(2)]_{p}=0.0107,} \\
{[G(2)]_{p}=[G(2)]_{n}} & =0.4159 . &
\end{array}
$$


When evaluating spin dependent cross-sections we use the following parameters for nuclear axial vector currents $[27,54]$

$$
\begin{array}{lll}
\Delta u^{(p)}=0.84, & \Delta d^{(p)}=-0.43, & \Delta s^{(p)}=-0.09, \\
\Delta u^{(n)}=\Delta d^{(p)}, & \Delta d^{(n)}=\Delta u^{(p)}, & \Delta s^{(n)}=\Delta s^{(p)} .
\end{array}
$$

We approximate all of isotopes of Xenon, in the Xenon 1T experiment, to have a mass number and atomic number $A_{X e}=131$ and $Z_{X e}=54$ respectively.

Open Access. This article is distributed under the terms of the Creative Commons Attribution License (CC-BY 4.0), which permits any use, distribution and reproduction in any medium, provided the original author(s) and source are credited.

\section{References}

[1] J. Abdallah et al., Simplified models for dark matter searches at the LHC, Phys. Dark Univ. 9-10 (2015) 8 [arXiv:1506.03116] [INSPIRE].

[2] F.J. Petriello, S. Quackenbush and K.M. Zurek, The invisible $Z^{\prime}$ at the CERN LHC, Phys. Rev. D 77 (2008) 115020 [arXiv:0803.4005] [INSPIRE].

[3] Y. Bai, P.J. Fox and R. Harnik, The Tevatron at the frontier of dark matter direct detection, JHEP 12 (2010) 048 [arXiv: 1005.3797] [INSPIRE].

[4] G. Busoni et al., Recommendations on presenting LHC searches for missing transverse energy signals using simplified s-channel models of dark matter, arXiv:1603.04156 [INSPIRE].

[5] LHC DARK MATter WORKING GRoup collaboration, LHC dark matter working group: next-generation spin-0 dark matter models, arXiv:1810.09420 [INSPIRE].

[6] H. Dreiner, D. Schmeier and J. Tattersall, Contact interactions probe effective dark matter models at the LHC, EPL 102 (2013) 51001 [arXiv: 1303.3348] [INSPIRE].

[7] A. Albert et al., Recommendations of the LHC dark matter working group: comparing LHC searches for heavy mediators of dark matter production in visible and invisible decay channels, arXiv:1703.05703 [INSPIRE].

[8] S. Chang, R. Edezhath, J. Hutchinson and M. Luty, Effective WIMPs, Phys. Rev. D 89 (2014) 015011 [arXiv: 1307.8120] [INSPIRE].

[9] H. An, L.-T. Wang and H. Zhang, Dark matter with t-channel mediator: a simple step beyond contact interaction, Phys. Rev. D 89 (2014) 115014 [arXiv:1308.0592] [INSPIRE].

[10] Y. Bai and J. Berger, Fermion portal dark matter, JHEP 11 (2013) 171 [arXiv:1308.0612] [INSPIRE].

[11] A. DiFranzo, K.I. Nagao, A. Rajaraman and T.M.P. Tait, Simplified models for dark matter interacting with quarks, JHEP 11 (2013) 014 [Erratum ibid. 01 (2014) 162] [arXiv: 1308.2679] [INSPIRE].

[12] N.F. Bell, Y. Cai, J.B. Dent, R.K. Leane and T.J. Weiler, Dark matter at the LHC: effective field theories and gauge invariance, Phys. Rev. D 92 (2015) 053008 [arXiv:1503.07874] [INSPIRE]. 
[13] A. Ibarra and S. Wild, Dirac dark matter with a charged mediator: a comprehensive one-loop analysis of the direct detection phenomenology, JCAP 05 (2015) 047 [arXiv:1503.03382] [INSPIRE].

[14] M. Garny, A. Ibarra and S. Vogl, Signatures of Majorana dark matter with t-channel mediators, Int. J. Mod. Phys. D 24 (2015) 1530019 [arXiv:1503.01500] [inSPIRE].

[15] P. Ko, A. Natale, M. Park and H. Yokoya, Simplified DM models with the full SM gauge symmetry: the case of t-channel colored scalar mediators, JHEP 01 (2017) 086 [arXiv: 1605. 07058] [INSPIRE].

[16] R.M. Godbole, G. Mendiratta and T.M.P. Tait, A simplified model for dark matter interacting primarily with gluons, JHEP 08 (2015) 064 [arXiv:1506.01408] [INSPIRE].

[17] Y. Bai and J. Osborne, Chromo-Rayleigh interactions of dark matter, JHEP 11 (2015) 036 [arXiv: 1506.07110] [INSPIRE].

[18] R.M. Godbole, G. Mendiratta, A. Shivaji and T.M.P. Tait, Mono-jet signatures of gluphilic scalar dark matter, Phys. Lett. B 772 (2017) 93 [arXiv: 1605.04756] [INSPIRE].

[19] G. D'Ambrosio, G.F. Giudice, G. Isidori and A. Strumia, Minimal flavor violation: an effective field theory approach, Nucl. Phys. B 645 (2002) 155 [hep-ph/0207036] [INSPIRE].

[20] A. Denner, H. Eck, O. Hahn and J. Kublbeck, Compact Feynman rules for Majorana fermions, Phys. Lett. B 291 (1992) 278 [INSPIRE].

[21] A. Denner, H. Eck, O. Hahn and J. Kublbeck, Feynman rules for fermion number violating interactions, Nucl. Phys. B 387 (1992) 467 [INSPIRE].

[22] J. Hisano, K. Ishiwata and N. Nagata, Gluon contribution to the dark matter direct detection, Phys. Rev. D 82 (2010) 115007 [arXiv:1007.2601] [INSPIRE].

[23] R.J. Hill and M.P. Solon, Standard Model anatomy of WIMP dark matter direct detection I: weak-scale matching, Phys. Rev. D 91 (2015) 043504 [arXiv:1401.3339] [INSPIRE].

[24] G. Jungman, M. Kamionkowski and K. Griest, Supersymmetric dark matter, Phys. Rept. 267 (1996) 195 [hep-ph/9506380] [INSPIRE].

[25] F. D'Eramo and M. Procura, Connecting dark matter UV complete models to direct detection rates via effective field theory, JHEP 04 (2015) 054 [arXiv: 1411.3342] [INSPIRE].

[26] R.J. Hill and M.P. Solon, Standard Model anatomy of WIMP dark matter direct detection II: QCD analysis and hadronic matrix elements, Phys. Rev. D 91 (2015) 043505 [arXiv: 1409.8290] [INSPIRE].

[27] M. Freytsis and Z. Ligeti, On dark matter models with uniquely spin-dependent detection possibilities, Phys. Rev. D 83 (2011) 115009 [arXiv:1012.5317] [INSPIRE].

[28] PICO collaboration, Dark matter search results from the PICO-60 $C_{3} F_{8}$ bubble chamber, Phys. Rev. Lett. 118 (2017) 251301 [arXiv: 1702.07666] [INSPIRE].

[29] SuperCDMS collaboration, Low-mass dark matter search with CDMSlite, Phys. Rev. D 97 (2018) 022002 [arXiv:1707.01632] [INSPIRE].

[30] LUX collaboration, Limits on spin-dependent WIMP-nucleon cross section obtained from the complete LUX exposure, Phys. Rev. Lett. 118 (2017) 251302 [arXiv:1705.03380] [INSPIRE].

[31] XENON collaboration, First dark matter search results from the XENON1T experiment, Phys. Rev. Lett. 119 (2017) 181301 [arXiv:1705. 06655] [INSPIRE]. 
[32] J. Alwall et al., The automated computation of tree-level and next-to-leading order differential cross sections and their matching to parton shower simulations, JHEP 07 (2014) 079 [arXiv: 1405.0301] [INSPIRE].

[33] A. Alloul, N.D. Christensen, C. Degrande, C. Duhr and B. Fuks, FeynRules $2.0-a$ complete toolbox for tree-level phenomenology, Comput. Phys. Commun. 185 (2014) 2250 [arXiv: 1310.1921] [INSPIRE].

[34] G. Ossola, C.G. Papadopoulos and R. Pittau, Reducing full one-loop amplitudes to scalar integrals at the integrand level, Nucl. Phys. B 763 (2007) 147 [hep-ph/0609007] [InSPIRE].

[35] C. Degrande, Automatic evaluation of $U V$ and $R_{2}$ terms for beyond the Standard Model Lagrangians: a proof-of-principle, Comput. Phys. Commun. 197 (2015) 239 [arXiv: 1406.3030] [INSPIRE].

[36] S. Dulat et al., New parton distribution functions from a global analysis of quantum chromodynamics, Phys. Rev. D 93 (2016) 033006 [arXiv: 1506.07443] [InSPIRE].

[37] ATLAS collaboration, Search for dark matter and other new phenomena in events with an energetic jet and large missing transverse momentum using the ATLAS detector, JHEP 01 (2018) 126 [arXiv: 1711.03301] [INSPIRE].

[38] CMS collaboration, Search for supersymmetry in multijet events with missing transverse momentum in proton-proton collisions at 13 TeV, Phys. Rev. D 96 (2017) 032003 [arXiv: 1704.07781] [INSPIRE].

[39] B. Dumont et al., Toward a public analysis database for LHC new physics searches using MadAnalysis 5, Eur. Phys. J. C 75 (2015) 56 [arXiv:1407.3278] [InSPIRE].

[40] D. Sengupta, The MadAnalysis5 implementation of the ATLAS in monojet+missing energy, (2018) [INSPIRE].

[41] M. Cacciari, G.P. Salam and G. Soyez, FastJet user manual, Eur. Phys. J. C 72 (2012) 1896 [arXiv:1111.6097] [INSPIRE].

[42] DELPHES 3 collaboration, DELPHES 3, a modular framework for fast simulation of a generic collider experiment, JHEP 02 (2014) 057 [arXiv: 1307.6346] [INSPIRE].

[43] T. Sjöstrand et al., An introduction to PYTHIA 8.2, Comput. Phys. Commun. 191 (2015) 159 [arXiv: 1410.3012] [INSPIRE].

[44] S. Höche et al., Matching parton showers and matrix elements, in HERA and the LHC. A workshop on the implications of HERA for LHC physics: proceedings part A, CERN-2005-014, (2005), pg. 288 [hep-ph/0602031] [INSPIRE].

[45] CMS collaboration, Search for supersymmetry in multijet events with missing transverse momentum in proton-proton collisions at $13 \mathrm{TeV}$, CMS-SUS-16-033, CERN, Geneva, Switzerland (2017).

[46] G. Bélanger, F. Boudjema, A. Goudelis, A. Pukhov and B. Zaldivar, MicrOMEGAs 5.0: freeze-in, Comput. Phys. Commun. 231 (2018) 173 [arXiv:1801.03509] [InSPIRE].

[47] SDM website, http://hep.pa.msu.edu/sdm/.

[48] T. Hahn, Generating Feynman diagrams and amplitudes with FeynArts 3, Comput. Phys. Commun. 140 (2001) 418 [hep-ph/0012260] [INSPIRE].

[49] T. Hahn and M. Pérez-Victoria, Automatized one loop calculations in four-dimensions and D-dimensions, Comput. Phys. Commun. 118 (1999) 153 [hep-ph/9807565] [InSPIRE]. 
[50] H.H. Patel, Package-X: a Mathematica package for the analytic calculation of one-loop integrals, Comput. Phys. Commun. 197 (2015) 276 [arXiv:1503.01469] [INSPIRE].

[51] V.A. Novikov, M.A. Shifman, A.I. Vainshtein and V.I. Zakharov, Calculations in external fields in quantum chromodynamics. Technical review, Fortsch. Phys. 32 (1984) 585 [INSPIRE].

[52] V. Shtabovenko, R. Mertig and F. Orellana, New developments in FeynCalc 9.0, Comput. Phys. Commun. 207 (2016) 432 [arXiv:1601.01167] [INSPIRE].

[53] Particle Data Group collaboration, Review of particle physics, Phys. Rev. D 98 (2018) 030001 [INSPIRE].

[54] Particle Data Group collaboration, Review of particle physics, J. Phys. G 37 (2010) 075021 [INSPIRE]. 